\title{
Drug targeting to sites of oxidative stress using the Baeyer-Villiger reaction
}

Thomas D. Avery ${ }^{1, a}$, Jiahe Li ${ }^{2, a}$, Dion J. L. Turner ${ }^{1}$, Fisher R. Cherry ${ }^{2}$, Mohd S. Ur Rasheed $^{2}$, Clarissa Aguilar ${ }^{2}$, Andrew J. Shepherd ${ }^{2}$, Jingxian $\mathrm{Yu}^{1}$, Peter M. Grace ${ }^{2 \star}$, Andrew D. Abell ${ }^{1 *}$.

${ }^{1}$ ARC Centre of Excellence for Nanoscale BioPhotonics (CNBP), Institute for Photonics and Advanced Sensing, Department of Chemistry, The University of Adelaide, Australia ${ }^{2}$ Laboratories of Neuroimmunology, Department of Symptom Research, and the MD Anderson Pain Research Consortium, University of Texas MD Anderson Cancer Center, Houston, USA

${ }^{a}$ Authors contributed equally

\section{${ }^{*}$ Correspondence:}

Andrew D. Abell, PhD

T: +61883135652

E: andrew.abell@adelaide.edu.au

Peter M. Grace, PhD

T: +1 7137926673

E:pgrace@mdanderson.org 


\section{ABSTRACT}

The antioxidant nuclear factor erythroid 2-related factor 2 (Nrf2) is a desirable therapeutic target for a broad range of pathologies, including chronic diseases of the lung and liver, and autoimmune, neurodegenerative, and cardiovascular disorders. However, current Nrf2 activators are limited by unwanted effects due to non-specificity, and systemic distribution and action. Here we report that a 1,2-dicarbonyl moiety masks the electrophilic reactivity of the Nrf2 activator monomethyl fumarate (MMF), otherwise responsible for its non-specific effects. The 1,2-dicarbonyl compound is highly susceptible to Baeyer-Villiger oxidation, with generation of MMF specifically on exposure to pathological levels of hydrogen peroxide or peroxynitrite. Oral treatment with the MMF generating 1,2-dicarbonyl compound reversed chronic neuropathic and osteoarthritis pain in mice, and selectively activated Nrf2 at sites of oxidative stress.

This 1,2-dicarbonyl platform may be used to treat additional disorders of oxidative stress, and to selectively target other therapeutics to sites of redox imbalance.

Keywords: nerve injury, chemotherapy-induced peripheral neuropathy 


\section{INTRODUCTION}

The ubiquity of oxidative stress as a pathological process has driven recent therapeutic interest in the antioxidant "master regulator" nuclear factor erythroid 2-related factor 2 $(\mathrm{Nrf2})^{1-3}$. In preclinical models, small molecule Nrf2 activators alleviate neurodegenerative, cardiovascular, and other diseases, but unwanted effects have hampered clinical translation ${ }^{2-4}$. For example, prolonged Nrf2 activation with long-term systemic treatments can cause kidney and heart damage ${ }^{5,6}$. While electrophilic compounds activate Nrf2 by succinating thiols of Kelch like ECH-associated protein 1 (Keap1)—a protein that otherwise sequesters Nrf2 in the cytosol—such agents are also prone to adverse effects due to indiscriminate interactions with other protein thiols ${ }^{1-4}$.

We aimed to overcome these long-standing issues by creating a compound that would mask the unwanted electrophilic nature of the Nrf2 activator monomethyl fumarate (MMF) until selective generation of MMF at sites of oxidative pathology. We reasoned that these features would be conferred to MMF with the addition of a 1,2-dicarbonyl functional group; 1,2-dicarbonyl compounds are biologically compatible and substrates for Baeyer-Villiger oxidation by hydrogen peroxide or peroxynitrite ${ }^{7-9}$. Peroxides are an ideal trigger for localized MMF generation as their diffusion beyond sites of pathology is restricted by their inherent reactivity ${ }^{3,10}$. Here, we report realization of a MMF generating 1,2-dicarbonyl compound. After chemical synthesis and characterization, we used mouse models of chronic pain—conditions driven in part by localized overproduction of hydrogen peroxide and peroxynitrite ${ }^{10,11}$-to demonstrate therapeutic efficacy and 
preferential activation of Nrf2 at local sites of oxidative stress by this first-in-class oxidative pathology-activated drug.

\section{RESULTS}

\section{Synthesis of MMF releasing 1,2-dicarbonyl compounds}

As MMF is a carboxylic acid, we explored 1,2-diketones, a-keto amides and a-keto esters as 1,2-dicarbonyl masking groups. These biologically compatible functional groups are highly susceptible to the desired Baeyer-Villiger oxidation ${ }^{7,9}$, selectively reacting with hydrogen peroxide and peroxynitrite to form an anhydride that will hydrolyse, generating only MMF and acid 3 (Fig. 1a). Representatives of each 1,2dicarbonyl chemotype (1,2-diketone (1a), a-keto amide (1b), a-keto ester (1c)) were synthesised (Fig. 1a; Fig. S1a-c).

\section{MMF generation from 1,2-dicarbonyl compounds by peroxides}

Each chemotype representative was evaluated by HPLC for its ability to generate MMF on exposure to peroxides. Compounds 1a-c completely and quantitatively generated MMF within 30 min at $1 \mathrm{mM}$ concentration in $100 \mathrm{mM}$ phosphate buffer $(\mathrm{pH} 7.4)$ containing five molar excess hydrogen peroxide (Fig. S2a-c). Both $\mathbf{1 b}$ and 1c were rapidly hydrolysed to a-keto acid $\mathbf{1 d}$ (Fig. S2b,c), a 1,2-dicarbonyl compound also capable of undergoing the same Baeyer-Villiger like oxidative cleavage to generate $M_{M}{ }^{12} .1 d$ was synthesized, isolated, characterized, and underwent rapid conversion to MMF in hydrogen peroxide containing buffer (Fig. S2d). Compounds 1b-d exhibit broad peaks in the HPLC due to equilibration with their hydrated form (geminal diol). By 
contrast, 1a exists predominantly in its keto form under these conditions as determined by NMR, and as such has a much narrower peak shape in the HPLC traces (Supplementary Information; Tables S1-5).

The ability of the 1,2-dicarbonyl functional group to mask the electrophilicity and selectively generate MMF on exposure to peroxides in biological systems will be governed by a range of factors, including reaction rates with peroxides in cytosolic and extracellular fluids, and hydrolysis rates and the extent of hydration (geminal diol). Additional pharmacokinetic factors (e.g., membrane permeability and metabolism of the equilibrium components (Tables S1-6)) will also influence selective Nrf2 activation in the presence of peroxides. To gain initial insights into behaviour of the compounds in biological systems, we evaluated 1,2-dicarbonyl compounds 1a-c for enhanced Nrf2 activity in the presence of pathological peroxide concentrations. Nrf2 activity was measured using a reporter HEK293 cell assay in which luciferase transcription is under the control of antioxidant response elements, themselves activated by $\mathrm{Nrf} 2^{1,2}$. The ability of these compounds to activate Nrf2 was evaluated in vitro, with comparison made to MMF alone. As expected ${ }^{13,14}$, MMF increased Nrf2 activity $(P<0.001)$ and target gene expression above media control $(P<0.001)$, without enhancement by pathological concentrations of hydrogen peroxide or peroxynitrite ${ }^{15,16}$ (Fig. 1b-e).

Nrf2 activity induced by compound 1a in physiological media was substantially lower than MMF (Fig. 1b; $P<0.001$ ), consistent with reduced electrophilic reactivity. Although Nrf2 activity was enhanced by pathological concentrations of hydrogen peroxide or 
peroxynitrite $(P<0.001)$, activity remained substantially lower than that induced by MMF (Fig. 1b; $P<0.001$ ). This suggests that 1a converts poorly to MMF under the experimental conditions. In contrast, Nrf2 activity induced by compound 1b was significantly higher than MMF, indicating greater electrophilic reactivity. However, hydrogen peroxide or peroxynitrite did not selectively enhance $\operatorname{Nrf2}$ activation $(P=$ 0.288), and 1b was cytotoxic at higher concentrations (Fig. 1c; data not shown). This indicates that $\mathbf{1 b}$ is more electrophilic than MMF and converts poorly to MMF under experimental conditions. We predict that with these unwanted features, compound 1b would be prone to non-selective interactions with protein thiols, causing adverse effects.

Compound 1c selectively enhanced Nrf2 activity in the presence of pathological concentrations of hydrogen peroxide $(10 \mu \mathrm{M})$ and peroxynitrite $(20 \mu \mathrm{M})($ Fig. $1 \mathrm{~d}$; $P<$ $0.001)$, but not physiological concentrations of hydrogen peroxide $(1 \mu \mathrm{M})$ (Fig. S3a; $P=$ 0.088 ) or peroxynitrite $(2 \mu \mathrm{M})$ (Fig. S3b; $P=0.061)^{15,16}$. This suggests that $1 \mathrm{c}$ is less electrophilic than MMF and has the appropriate rate of MMF production under the experimental conditions to selectively enhance Nrf2 activity. Increased Nrf2 activity was accompanied by elevated expression of target genes Sod1 $(P=0.004), H \operatorname{mox} 1(P=$ $0.001)$, and $\operatorname{Gclm}(P=0.002)$ (Fig. 1e). We next tested whether the biological activity of 1c was driven by MMF using the 1,2-dicarbonyl a-keto ester compound $\mathbf{5 b}$ (dimethyl 2oxoglutarate) (Fig. S1a). Compound $\mathbf{5 b}$ is chemically similar to $\mathbf{1 c}$ in its reactivity to hydrogen peroxide (Table S5) and susceptibility to hydrolysis (Table S6), also generating metabolites methanol and carbon dioxide in addition to non-electrophilic, monomethyl succinate (saturated MMF). Monomethyl succinate lacks the $\alpha, \beta-$ 
unsaturated system of MMF and thus cannot succinate Keap1 thiols, a key step in activation of Nrf2 by MMF. Compound 5b failed to activate Nrf2 (Fig. S3c); with MMF being the only product absent after exposure of $\mathbf{5 b}$ to peroxides, this result critically confirms MMF as the therapeutic metabolite of 1c. Given selective activation of Nrf2 (on par with MMF) in the presence of pathological hydrogen peroxide and peroxynitrite, compound 1c (Fig. 1f) was selected for validation in mouse models of chronic pain.

\section{1,2-Dicarbonyl 1c reverses chronic pain}

Peripheral nerve injury can lead to neuropathic pain ${ }^{17}$, driven in part by localised increases in hydrogen peroxide and peroxynitrite that hyperexcite sensory neurons ${ }^{10,11}$. We therefore examined the anti-nociceptive efficacy of 1,2-dicarbonyl compound 1c in the spared nerve injury (SNI) model of neuropathic pain ${ }^{18}$ (Fig. 2a). Multi-day oral administration of compound 1c, beginning at peak SNI-induced pain (postoperative day 7), dose-dependently reversed mechanical allodynia (Fig. 2b, Fig. S4a, $P<0.001$ ) and dynamic allodynia as measured by changes in paw withdrawal responses (Fig. 2c, Fig. S4b, $P<0.001)$. Diroximel fumarate, FDA-approved prodrug that systemically distributes $\mathrm{MMF}^{4}$, also attenuated mechanical (Fig. 2b, $P<0.001$ ) and dynamic allodynia (Fig. 2c, $P<0.001$ ) at equi-mole doses. Treatment with compound $5 \mathbf{b}$, which generates saturated (non-electrophilic) MMF on exposure to peroxides, did not reverse SNI-induced allodynia (Fig. S4c, $P=0.864$ ). This result confirms MMF as the therapeutic metabolite of 1c in vivo. In sham-operated animals, the highest effective dose of compound 1c did not alter withdrawal responses to mechanical (Fig. S4a, $P=$ 0.139 ) or dynamic stimuli (Fig. S4b, $P=0.188$ ) compared to vehicle, suggesting that 1c 
does not alter normal sensory function. Dosing 1c for five consecutive days did not lead to tolerance or a loss of efficacy, since reversal of mechanical allodynia increased over time (Fig. 2d, $P<0.001$ ). In contrast, anti-nociceptive effects of morphine diminished when given over a similar timecourse (Fig. $2 \mathrm{~d}, P=0.025)$.

We further tested antinociceptive efficacy of compound 1c in a clinically relevant models of cisplatin chemotherapy-induced peripheral neuropathy (CIPN) ${ }^{19,20}$ and osteoarthritis (surgical destabilization of the medial meniscus $\left.(\mathrm{DMM})^{21}\right)$. Oxidative/nitrative species mediate in part cisplatin-CIPN and osteoarthritis pain ${ }^{22,23}$. When administered after the second cycle of cisplatin was complete, multi-day treatment with oral 1c reversed mechanical allodynia (Fig. S4d, $P<0.001$ ). We also assessed the effect of 1c on numbness, a common symptom of $\mathrm{CIPN}^{20}$, by placing an adhesive patch on the hind paw and recording the latency to attend to the patch ${ }^{19}$. Mice treated with 1c after the second cycle of cisplatin was complete had a faster response time (Fig. S4e, $P=$ 0.011), indicating reversed numbness compared to mice treated with vehicle. Treatment with compound 1c further reversed allodynia in the DMM model (Fig. S4f, $P=0.003$ ). These data demonstrate anti-nociceptive efficacy of compound 1c in preclinical chronic pain models of differing aetiologies, but with common underlying mechanisms of oxidative/nitrative stress.

In addition to stimulus-evoked pain, patients with chronic pain frequently experience ongoing (spontaneous/paroxysmal) pain ${ }^{17}$. We therefore assessed ongoing neuropathic pain using the conditioned place preference paradigm ${ }^{24}$, with the nerve blocker 
retigabine as the conditioning stimulus ${ }^{19,25}$. SNI-operated mice treated with vehicle spent more time in the retigabine-paired chamber, compared to sham-operated mice, indicating ongoing pain (Fig. 2e, $P=0.007$ ). Compare the vehicle treated SNI-operated mice, those treated with compound 1c had reduced time spent in the retigabine-paired chamber, indicating that compound 1c had reversed ongoing pain (Fig. 2e, $P<0.001$ ). Equi-mole diroximel fumarate treatment similarly reversed ongoing pain (Fig. 2e, $P=$ $0.018)$.

\section{Site selective Nrf2 activation by 1,2-dicarbonyl $1 \mathrm{c}$}

Nrf2 activation was monitored as a proxy for site specific generation of MMF from compound 1c in the SNI model. We first validated Nrf2 as a therapeutically-relevant biomarker by confirming that the anti-nociceptive effects of compound 1c were dependent on Nrf2, as for other MMF-releasing compounds ${ }^{26}$. When orally administered to SNI-operated mice deficient in $\mathrm{Nrf2}\left(\mathrm{Nfe} 2 \mathrm{I}^{--}\right)$, 1c failed to reverse mechanical allodynia, compared to littermate wildtype controls (Fig. 2f, $P<0.001$ ). There were no genotype differences in baseline thresholds or acute morphine analgesia (Fig. S4g,h; $P$ $=0.597$ (mechanical allodynia); $P=0.584$ (dynamic allodynia)), excluding genotype as a confounding variable. These data indicate that Nrf2 mediates the antinociceptive effects of compound 1c.

Unilateral peripheral nerve injury induces oxidative/nitrative stress in the ipsilateral sciatic nerve and $L 4 / 5$ dorsal root ganglia (DRG; Fig. 2a) ${ }^{10,11,27}$. We tested whether 1c would exclusively activate Nrf2 in these tissues as an indicator of selective cleavage of 
MMF in response to local oxidative/nitrative stress. Compound 1c induced Nrf2 nuclear translocation (activation) only in the sciatic nerve injury site (Fig. S5a, $P<0.001$ ) and ipsilateral DRG (Fig. 2g, $P<0.001$ ), accompanied by selectively increased expression of Nrf2 target antioxidant genes in the ipsilateral DRG (Fig. 2h). In contrast, diroximel fumarate non-selectively activated Nrf2 and increased expression of antioxidant genes in the contralateral tissues as well (Fig. 2g, Fig. S5a, $P<0.001$ ). Further demonstrating site-selective activation in contrast to diroximel fumarate, compound 1c did not induce Nrf2 nuclear translocation at other unwanted sites (Fig. S5b; liver $(P=0.663)$, kidney $(P$ $=0.909)$, lung $(P=0.999)$. Compound $\mathbf{5 b}$ did not increase nuclear levels of Nrf2 in ipsilateral DRG, confirming that MMF generation by 1c was responsible for Nrf2 translocation (Fig. S5c; $P=0.999$ ).

Leukopenia is a common unwanted effect related to the systemic distribution of $\mathrm{MMF}^{4,28}$. We assessed whether administration of compound $1 \mathrm{c}$ would have reduced immunosuppressive consequences as further evidence of spatially restricted pharmacodynamics. Diroximel fumarate reduced the total leukocyte count, as expected (Fig. 2i; $P=0.039$ ). However, oral treatment with $1 \mathrm{c}$ did not reduce total leukocyte count, compared to mice treated with vehicle $(P=0.651)$, indicating reduced immunosuppression.

\section{DISCUSSION}

We have leveraged the Baeyer-Villiger chemical reaction in a biological setting to selectively deliver a small molecule Nrf2 activator in response to localized oxidative 
stress. Masking MMF with a 1,2-dicarbonyl moiety to allow generation at sites of pathology addresses the long-standing issue of side effects caused by systemic distribution of electrophilic drugs ${ }^{2,3}$. Importantly, 1,2-dicarbonyl compound 1c is only cleaved by pathological concentrations of hydrogen peroxide or peroxynitrite, which is expected to leave physiological redox signalling intact ${ }^{29}$. The selective cleavage activates Nrf2 at sites of pathology in vivo, which mediates anti-nociceptive efficacy. Compound 1c could also exert therapeutic effects by scavenging peroxides when participating in Baeyer-Villiger oxidation.

Compound 1c and other 1,2-dicarbonyl compounds are suitable drug candidates, as they have broad synthetic scope to optimize pharmacokinetic and toxicological properties. The diversity offers further advantage in that the cleaved carboxylic, carbonic, or carbamic acid fragment 3 (Fig. 1a) can be relatively low in molecular weight and selected to be biologically benign and non-toxic.

We show that oral delivery of compound 1c to activate Nrf2 at local sites of oxidative stress reverses chronic pain of diverse aetiologies in preclinical models, consistent with Nrf2-dependent antinociceptive effects of fumarates ${ }^{26}$. Nrf2 activation is an attractive therapeutic target because its activation increases levels of the panoply of endogenous antioxidants that scavenge free radicals and their precursors, which underlie chronic pain pathology ${ }^{10,11,27}$. Activating Nrf2 with a single small molecule is therefore superior to supplementation of individual antioxidants that generally have unfavorable pharmacokinetic and pharmacodynamic profiles ${ }^{3,10,11}$. Chronic pain remains a large 
unmet medical need, and non-addictive treatments that engage endogenous pain resolution mechanisms like Nrf2 are a unique approach ${ }^{30}$.

Oxidative stress is a pathological process common to numerous diseases and disorders $^{1-3} \cdot 1,2$-Dicarbonyl 1c therefore has potential to treat a wide range of pathologies. The 1,2-dicarbonyl platform could further have broad application in targeting other molecular payloads to sites of oxidative stress.

\section{METHODS}

\section{1,2-Dicarbonyl compound synthesis and characterisation}

All anhydrous solvents were commercially obtained and stored in Sure-Seal bottles under nitrogen or transferred to an Inert Corporation Solvent Purification System and dispensed from there. All other reagents and solvents were purchased as the highest grade required and used without further purification. All organic extracts were dried over anhydrous magnesium sulfate $\left(\mathrm{MgSO}_{4}\right)$. Thin-layer chromatography (TLC) used aluminum sheets coated with silica gel $60 \mathrm{~F}_{254}$ from Merck and were visualized using ultraviolet light. Melting points were taken on a Reichert Thermovar Kofler apparatus and are uncorrected. Infrared spectra were recorded on a Perkin Elmer Spectrum 400 FT-IR / FT-FIR Spectrometer as neat samples unless otherwise stated. ${ }^{1} \mathrm{H}$ NMR and ${ }^{13} \mathrm{C}$ NMR, spectra were acquired on an Agilent $500 \mathrm{MHz}$ spectrometer or Agilent 600 $\mathrm{MHz}$ spectrometer. High resolution mass spectrometry (HRMS) was performed on an Agilent 6230 ESI-TOF LCMS. Analytical RP HPLC spectra were acquired on an Agilent 1100 Infinity series machine using a Phenomenex Luna C18(2) column (250 × 4.6 mm, 
$100 \AA, 5 \mu \mathrm{m}$ ) with a buffer system of $0.1 \%$ TFA in water (buffer $A$ ) and $0.1 \%$ TFA in acetonitrile (buffer B), elution was with a gradient of $0-100 \%$ A-B over 20 min. All yields reported refer to isolated material judged to be homogeneous by TLC, HPLC and NMR spectroscopy.

a-Keto amide $\mathbf{1 b}$ was synthesized as reported ${ }^{31}$ and $\mathbf{a - k e t o}$ ester $\mathbf{1 c}$ was synthesized from dimethyl 2-oxoglutarate $\mathbf{5 b}$, using the similar bromination / elimination conditions described for $\mathbf{1 b}$ (Fig. S1a). Both compounds were stored as solids at $4^{\circ} \mathrm{C}$ in the absence of light to avoid UV light assisted isomerization.

We designed a synthetic approach to aromatic 1,2-diketones of type 1a (Fig. S1b). trans-Cinnamaldehyde 6 reacted with stabilized phosphorus ylide 7 to give trans, trans 1,3-butadiene $\mathbf{8}$ as the major product. Purified diene $\mathbf{8}$ was then selectively dihydroxylated employing standard Sharpless conditions outlined for closely related compounds $^{32}$. 1,2-Diketone $\mathbf{1 a}$ was generated by treatment of purified diol $\mathbf{1 0}$ with Dess-Martin periodinane, neat. This technique was used to minimize competing oxidative cleavage of the diol to benzaldehyde and methyl (2E)-4-oxo-2-butenoate. 1,2Diketone 1a was found to isomerise to the cis isomer in deuterated chloroform on exposure to ambient light. Once solvent was removed and 1a was stored without solvent it reverted to and remained as the trans isomer. 1,2-Diketone 1a was fully characterized and its identity confirmed by subsequent reaction with hydrogen peroxide to generate benzoic acid and MMF. The IR and ${ }^{1} \mathrm{H}$ NMR spectra we obtained did not match those reported in the literature (Tables S3), indicating 1a was not the product 
obtained from the high-pressure palladium catalysed double carbonylative vinylation of 4-iodobenzene and methyl acrylate in the presence of carbon monoxide, as reported ${ }^{33}$. Ours is therefore the first reported synthesis of 1,2-diketones of type 1a (Fig. S1b).

a-Keto acid 1d was synthesised from 1c obtaining it pure by extraction from its hydrolysis reaction in $100 \mathrm{mM}$ phosphate buffer (pH7.4).

\section{In vitro Nrf2 reporter assay}

NRF2/ARE luciferase reporter HEK293 cells (SL-0042-NP, Signosis, Santa Clara, USA) were maintained in Dulbecco's Modified Eagle's Medium (DMEM) (SH30243.01, Cytiva, Marlborough, USA) supplemented with 10\% Fetal Bovine Serum (FBS) (03-600-511, ThermoFisher Scientific, Waltham, USA) and 1\% Penicillin/Streptomycin (SV30010, Cytiva). Cells were seeded into 48-well flat bottom cell culture plate (3548, Corning, Corning, USA) in $500 \mu \mathrm{L}$ of supplemented DMEM at a concentration of $2 \times 10^{5} \mathrm{cells} / \mathrm{mL}$ and incubated overnight at $37^{\circ} \mathrm{C}$ with $5 \% \mathrm{CO}_{2}$ in a humidified environment. Prior to treatment, the media was replaced with fresh $0.1 \%$ FBS in DMEM. TertButylhydroquinone (tBHQ; 0, 0.5-30 $\mu \mathrm{M}$; 112941, Sigma-Aldrich, St Louis, USA), a wellknown Nrf2 activator, was used as a positive control to confirm Nrf2-dependent luciferase production (Fig. S6). To identify concentrations of hydrogen peroxide and peroxynitrite that did not activate Nrf2 per se, cells were first treated with concentration ranges of hydrogen peroxide (H1009, Sigma-Aldrich) or peroxynitrite (20-107, SigmaAldrich) (0, 1-300 $\mu \mathrm{M})$ (Fig. S5). In the next series of experiments, cells were treated with concentration ranges of $\mathbf{1 a}$ (synthesized by T. Avery), $\mathbf{1 b}$ (synthesized by T. 
Avery), 1c (synthesized by T. Avery), 5b (349631, Sigma-Aldrich), or monomethyl fumarate (651419, Sigma-Aldrich) (0,1-100 $\mu \mathrm{M})$, followed by a fixed concentrations of hydrogen peroxide (1 or $10 \mu \mathrm{M})$, peroxynitrite (1 or $20 \mu \mathrm{M})$, or media control. These concentrations respectively represent physiological and pathological levels ${ }^{15,16}$. Cells were incubated with the treatments for 16 hours. After washing with phosphate buffer saline (PBS) (ThermoFisher Scientific), cells were lysed by a 15-min incubation at room temperature with passive lysis buffer (E1941, Promega, Madison, USA). Cell lysates (30 $\mu \mathrm{L})$ were transferred to 96-well white/clear flat bottom plate (3632, Corning), and mixed with $150 \mu \mathrm{L}$ of luciferase substrate (LUC100, Signosis). The plates were read in a Synergy HTX Multi-Mode Reader (BioTek, Winooski, USA). All conditions were performed in triplicate.

\section{Animals}

Pathogen-free adult male and female C57BL/6J mice (8 weeks old on arrival; The Jackson Laboratory, Bar Harbor, USA) were used. Male and female (8 to 12 weeks old) wild type and $\mathrm{Nfe} 2 / 2^{/-}$mice on a C57BL/6J genetic background (The Jackson Laboratory) were bred at The University of Texas MD Anderson Cancer Center (Houston, USA). Mice were housed five per cage in a light- and temperature-controlled room (12:12-h light-dark cycle, light on at 7:00 AM) with food and water available ad libitum. All procedures were approved by the MD Anderson Cancer Center Animal Care and Use Committee.

\section{Spared nerve injury (SNI) surgery}


$\mathrm{SNI}^{18}$ was performed as described for mice ${ }^{34}$. Briefly, under inhaled isoflurane anesthesia, the tibial and common peroneal nerves were isolated, tightly ligated with 6-0 silk (707G, Ethicon, Somerville, USA), and transected immediately distal to the ligation. The sural nerve was left intact. For sham surgery, the nerves were exposed, but not ligated or transected. Animals were monitored post-operatively until fully ambulatory prior to return to their home cage.

\section{Surgical destabilization of the medial meniscus (DMM)}

Mice were subjected to the DMM model of osteoarthritis in one hindlimb, as previously described ${ }^{21}$. Mice were anesthetized with isoflurane and placed in dorsal recumbency. Carprofen (5mg/kg, SQ) was administered immediately prior to beginning surgery. A 3 $\mathrm{mm}$ longitudinal incision was made over the right patella, and the joint capsule was opened with micro Vannas scissors under $4 \mathrm{X}$ magnification. Blunt dissection of the infrapatellar fat pad exposed the meniscotibial ligament of the medial meniscus, which was then transected using a \#11 scalpel blade. The incision was closed with two $9 \mathrm{~mm}$ AutoClip wound clips, which were removed 10 days post-surgery. Sham surgery consisted of the same anesthesia, analgesia and skin/joint capsule incisions, without transection of the meniscotibial ligament. Animals were monitored post-operatively until fully ambulatory prior to return to their home cage.

\section{In vivo drug treatments}

Cisplatin (TEVA Pharmaceuticals, North Wales, PA) was diluted in sterile saline and administered for 5 days (2.3 mg/kg/day, i.p.) followed by 5 days of rest and a second 
round of 5 doses to induce chemotherapy-induced neuropathy (CIPN) ${ }^{19}$. Compound 1c $(\mathrm{MW}=172.14)$ and diroximel fumarate $(\mathrm{MW}=255.23)$ (synthesized by $\mathrm{T}$. Avery) were suspended in methylcellulose (viscosity $15 \mathrm{cP}, 2 \% \mathrm{w} / \mathrm{v}$ in water; Sigma-Aldrich). For SNI anti-nociception: 1c (100, 225, or $350 \mu \mathrm{mol} / \mathrm{kg} /$ day, p.o.), 1 d (350 $\mu \mathrm{mol} / \mathrm{kg} /$ day, p.o.), diroximel fumarate $(350 \mu \mathrm{mol} / \mathrm{kg} /$ day, p.o.) or methylcellulose or phosphate buffer vehicle controls (equivolume, p.o.) were administered daily, beginning 7 days after SNI/sham, and continuing for 3 (reflex tests) or 7 days (conditioned place preference tests). For CIPN anti-nociception: 1c (350 $\mu \mathrm{mol} / \mathrm{kg} /$ day, p.o.) or methylcellulose vehicle were administered for 5 consecutive days, beginning 3 days after the last cisplatin dose. For anti-nociceptive tolerance: 1c (350 $\mu \mathrm{mol} / \mathrm{kg} /$ day, p.o.) or the positive control morphine sulfate (6 mg/kg/day, s.c.; gifted from the National Institute on Drug Abuse Drug Supply Program, Research Triangle Institute, NC) were administered daily for 5 days, beginning 7 days after SNI. For acute morphine analgesia: morphine sulfate (5 mg/kg, s.c.; gifted from the National Institute on Drug Abuse Drug Supply Program). For leukopenia assessment: naïve mice were treated with 1c (350 $\mu \mathrm{mol} / \mathrm{kg} /$ day, p.o.), diroximel fumarate (350 $\mu \mathrm{mol} / \mathrm{kg} /$ day, p.o.), or methylcellulose vehicle were administered daily for 10 days.

\section{Behavioural sensory testing}

All behavioural tests were conducted by an experimenter who was blinded to group assignments. Mice received at least three 60-min habituations to the test environment before reflex testing. Rodents were placed in a small plexiglass enclosure on a mesh stand. Tactile allodynia was measured using the von Frey test as described 
previously $^{26}$. The $50 \%$ paw withdrawal threshold was determined using the "up-down" method $^{35}$.

Dynamic allodynia was measured by lightly stroking the plantar surface of the hindpaw with a soft paintbrush, using a protocol and scoring system developed by Dr. Enrique José Cobos (personal communication), as previously reported ${ }^{36}$. A paintbrush (5/0, Princeton Art \& Brush Co.) was prepared by blunting the tip and removing the outer layer of hairs. The lateral plantar region of the left hindpaw (sural nerve territory) was stimulated by light stroking $(\sim 2 \mathrm{~cm} / \mathrm{s})$ with the paintbrush, in the direction from heel to toe. The paw withdrawal response was scored according to the following criteria, score $=0$ : walking away or occasionally very brief paw lifting $(\leq 1 \mathrm{~s})$; score $=1$ : sustained lifting (>2s) of the stimulated paw toward the body; score = 2: a strong lateral lifting above the level of the body; score = 3: flinching or licking of the affected paw. Average scores for each mouse were obtained from three stimulations at intervals of at least 3 $\min$.

Spontaneous pain was tested using a conditioning paradigm with retigabine (\#R-100; Alomone Laboratory, Jerusalem, Israel) as the conditioned stimulus to briefly relieve pain, as previously described ${ }^{19,25,37}$. Mice were first allowed to freely explore the conditioned place preference apparatus, consisting of two chambers (one dark, one light) connected by a hallway (Stoelting, Wood Dale, USA), for 15 minutes. The time spent in the light chamber was recorded. During the conditioning phase, mice were first administered saline (i.p.) and kept in the dark chamber for 20 minutes. Three hours 
later, the analgesic retigabine was administered (10 mg/kg; i.p.) and after $10 \mathrm{mins}$, the mice were placed in the light chamber for 20 minutes. The conditioning was completed over four consecutive days. On the fifth day, the mice were again allowed to freely explore the apparatus for 15 minutes without any retigabine/saline injections. Data are presented as the difference in time spent in the light (retigabine-paired) chamber during the drug-free test on day five minus time spent in the light chamber at baseline (preconditioning phase). A mouse with spontaneous pain should show an increase in time spent in the light chamber that was paired with retigabine than it did in the preconditioning phase.

To examine numbness, we used a modified protocol of the adhesive removal test, as described ${ }^{19,38}$. Briefly, a round adhesive patch (3/16" Teeny Tough-Spots; USA Scientific INC, Ocala, USA) was placed on the plantar surface of the hind paws. The latency to attend to the patch (e.g., shaking or attempted removal) was recorded within a 15-minute testing time.

\section{Tissue collection}

Within $4 \square \mathrm{h}$ of the final dose, mice were deeply anesthetized with Beuthanasia-D (Merck, Kenilworth, USA) and then transcardially perfused with ice-cold saline. In some experiments, blood was collected via cardiac puncture prior to perfusion. The ipsilateral and contralateral sciatic nerve ( $5 \mathrm{~mm}$ proximal to the transection), L4/5 dorsal root ganglia (DRG), liver, kidney, and lung were isolated and rapidly frozen for subsequent analysis. 


\section{qPCR}

Total RNAs were extracted from HEK293 cells or DRG tissues using TRIzol

(ThermoFisher Scientific). One $\mu \mathrm{g}$ RNA was used for reverse transcription with iScript Reverse Transcription Supermix (Bio-Rad). Real-time polymerase chain reaction was carried out in a final volume of $20 \mu$ with iTaq Universal SYBR Green Supermix (BioRad) containing $2 \mu$ of five times diluted cDNA and monitored by CFX Connect RealTime PCR Detection System (Bio-Rad). The following cycling parameters were used: $95^{\circ} \mathrm{C}$ for $3 \mathrm{~min}, 40$ cycles of $95^{\circ} \mathrm{C}$ for $5 \mathrm{~s}$, and $60^{\circ} \mathrm{C}$ for $30 \mathrm{~s}$. Primer sequences are reported in Table S7. The level of the target mRNA was quantified relative to the housekeeping gene (Gapdh) using the $\Delta \Delta \mathrm{CT}$ method. Gapdh was not significantly different between treatments.

\section{Western blotting}

DRG from 3 mice or sciatic nerves from two mice were pooled within groups (treatment, lateralization, sex) to ensure that sufficient protein could be obtained for analysis. Liver, kidney, and lung were not pooled. Nuclear fractions were isolated with a NE-PER Nuclear and Cytoplasmic Extraction Kit (78835, ThermoFisher Scientific), according to manufacturer instructions. Western blotting was performed as previously described ${ }^{3}$. Nuclear proteins were subjected to NuPAGE Bis-Tris (4 to 12\%) gel electrophoresis under reducing conditions. After transfer to nitrocellulose membranes (IB23001, Invitrogen, USA), non-specific binding sites were blocked with Superblock buffer (37515, ThermoFisher Scientific) for 1 hour at room temperature. Membranes were 
incubated overnight at $4^{\circ} \mathrm{C}$ with primary anti-Nrf2 antibody $(1: 1000$; rabbit polyclonal IgG; ab31163, Abcam) and anti-histone H3 antibody (1:2000; rabbit polyclonal IgG; ab1791, Abcam) (loading control). The membranes were then washed with phosphate buffered saline containing $0.1 \%$ Tween-20 and probed with horseradish peroxidase secondary antibody (1:5,000; goat polyclonal IgG; Jackson ImmunoResearch, West Grove, USA) in blocking buffer containing $0.1 \%$ Tween-20 for $1 \square \mathrm{h}$ at room temperature. After washing with $1 \times$ PBS containing $0.1 \%$ Tween-20, membranes were developed with enhanced chemiluminescent substrate (ThermoFisher Scientific). Images were acquired using ImageQuant LAS 4000 (GE Healthcare Life Sciences, USA).

Densitometry analysis was performed using ImageQuant TL software (GE Healthcare Life Sciences). Data were normalized to loading control (histone H3).

\section{Leukocyte counting}

Leukocytes from cardiac blood were stained with Türk's solution (Sigma-Aldrich) according to manufacturer instructions, and manually counted on a hemocytometer by an experimenter who was blinded to treatment conditions.

\section{Statistics}

Differences between in vitro concentration-response relationships were determined by comparing the slopes of fitted functions for shared parameters. Linear models were selected as the pharmacologically-relevant concentrations tested were within the linear range of the concentration-response functions. Von Frey and brush data were analysed by repeated measures two- or three-way ANOVA with Tukey post hoc tests where 
appropriate. Conditioned place preference data were analysed by two-way ANOVA with Tukey post hoc tests. Data from the adhesive removal test were analysed by unpaired T test. Gene expression, protein levels, and leukocyte numbers were analysed by oneway ANOVA followed by Dunnett or Tukey post hoc tests as appropriate. Analyses were performed using Prism 9 (GraphPad). $P<0.05$ was considered statistically significant. Data are expressed as mean \pm SD.

\section{ACKNOWLEDGEMENTS}

This work is supported by National Institutes of Health grants RF1 NS113840 (P.M.G.), and in part by P30 CA016672 (MD Anderson Cancer Center); and, Australian Research Council grants CE140100003 and DP180101581 (A.D.A.).

\section{AUTHOR CONTRIBUTIONS}

T.D.A., P.M.G., and A.D.A. conceived the study. T.D.A, J.L., D.J.L.T., P.M.G., and A.D.A. designed most of the experiments, and A.J.S. and J.Y. designed certain experiments. T.D.A., J.L., D.J.L.T., F.R.C., M.S.U.R., C.A, J.Y. collected the data. T.D.A, P.M.G., and A.D.A. drafted the paper. All authors contributed to data analysis and writing of the paper.

\section{COMPETING INTERESTS}

T.D.A., J.L., D.J.L.T., P.M.G., and A.D.A. are named inventors on PCT patent application AU2021/050217 covering peroxide-activated prodrugs to treat disorders of 
bioRxiv preprint doi: https://doi.org/10.1101/2021.09.03.458872· this version posted September 6, 2021. The copyright holder for this preprint

(which was not certified by peer review) is the author/funder, who has granted bioRxiv a license to display the preprint in perpetuity. It is made available under aCC-BY-NC-ND 4.0 International license.

oxidative stress. T.D.A, P.M.G., and A.D.A. receive funding from Biogen Inc. The other authors declare no competing financial interests. 


\section{REFERENCES}

1 Dodson, M. et al. Modulating NRF2 in Disease: Timing Is Everything. Annu Rev Pharmacol Toxicol 59, 555-575 (2019).

2 Cuadrado, A. et al. Therapeutic targeting of the NRF2 and KEAP1 partnership in chronic diseases. Nat Rev Drug Discov 18, 295-317 (2019).

3 Forman, H. J. \& Zhang, H. Targeting oxidative stress in disease: promise and limitations of antioxidant therapy. Nat Rev Drug Discov (2021).

4 Hoogendoorn, A. et al. Emerging Therapeutic Applications for Fumarates. Trends Pharmacol Sci 42, 239-254 (2021).

5 de Zeeuw, D. et al. Bardoxolone methyl in type 2 diabetes and stage 4 chronic kidney disease. N Engl J Med 369, 2492-2503 (2013).

6 Zhao, S. et al. Nrf2 Deficiency Upregulates Intrarenal Angiotensin-Converting Enzyme-2 and Angiotensin 1-7 Receptor Expression and Attenuates Hypertension and Nephropathy in Diabetic Mice. Endocrinology 159, 836-852 (2018).

7 Abo, M. et al. Development of a Highly Sensitive Fluorescence Probe for Hydrogen Peroxide. J Am Chem Soc 133, 10629-10637 (2011).

8 Mallet, R. T., Sun, J., Knott, E. M., Sharma, A. B. \& Olivencia-Yurvati, A. H. Metabolic cardioprotection by pyruvate: Recent progress. Exp Biol Med 230, 435443 (2005).

9 Sawaki, Y. \& Foote, C. S. Acyclic Mechanism in the Cleavage of Benzils with Alkaline Hydrogen-Peroxide. J Am Chem Soc 101, 6292-6296 (1979).

10 Grace, P. M. et al. Nitroxidative Signaling Mechanisms in Pathological Pain. Trends in Neurosciences 39, 862-879 (2016).

11 Squillace, S. \& Salvemini, D. Nitroxidative stress in pain and opioid-induced adverse effects: therapeutic opportunities. Pain (2021).

12 Siegel, B. \& Lanphear, J. Kinetics and Mechanisms for the Acid-Catalyzed Oxidative Decarboxylation of Benzoylformic Acid. J Org Chem 44, 942-946 (1979).

13 Ahuja, M. et al. Distinct Nrf2 Signaling Mechanisms of Fumaric Acid Esters and Their Role in Neuroprotection against 1-Methyl-4-Phenyl-1,2,3,6-

Tetrahydropyridine-Induced Experimental Parkinson's-Like Disease. J Neurosci 36, 6332-6351 (2016).

14 Linker, R. A. et al. Fumaric acid esters exert neuroprotective effects in neuroinflammation via activation of the Nrf2 antioxidant pathway. Brain 134, 678$692(2011)$.

15 Forman, H. J., Bernardo, A. \& Davies, K. J. What is the concentration of hydrogen peroxide in blood and plasma? Arch Biochem Biophys 603, 48-53 (2016).

16 Radi, R. Oxygen radicals, nitric oxide, and peroxynitrite: Redox pathways in molecular medicine. Proc Natl Acad Sci U S A 115, 5839-5848 (2018).

17 Colloca, L. et al. Neuropathic pain. Nat Rev Dis Primers 3, 17002 (2017).

18 Decosterd, I. \& Woolf, C. J. Spared nerve injury: an animal model of persistent peripheral neuropathic pain. Pain 87, 149-158 (2000). 
19 Krukowski, K. et al. HDAC6 inhibition effectively reverses chemotherapy-induced peripheral neuropathy. Pain 158, 1126-1137 (2017).

20 Shah, A. et al. Incidence and disease burden of chemotherapy-induced peripheral neuropathy in a population-based cohort. J Neurol Neurosurg Psychiatry 89, 636-641 (2018).

21 Glasson, S. S., Blanchet, T. J. \& Morris, E. A. The surgical destabilization of the medial meniscus (DMM) model of osteoarthritis in the 129/SvEv mouse. Osteoarthritis Cartilage 15, 1061-1069 (2007).

22 Shim, H. S. et al. Peripheral and central oxidative stress in chemotherapyinduced neuropathic pain. Mol Pain 15, 1744806919840098 (2019).

23 Berenbaum, F. Osteoarthritis as an inflammatory disease (osteoarthritis is not osteoarthrosis!). Osteoarthritis Cartilage 21, 16-21 (2013).

24 King, T. et al. Unmasking the tonic-aversive state in neuropathic pain. Nat Neurosci 12, 1364-1366 (2009).

25 Yang, Q. et al. Persistent pain after spinal cord injury is maintained by primary afferent activity. J Neurosci 34, $10765-10769$ (2014).

$26 \mathrm{Li}, \mathrm{J}$. et al. Oral Dimethyl Fumarate Reduces Peripheral Neuropathic Pain in Rodents via NFE2L2 Antioxidant Signaling. Anesthesiology 132, 343-356 (2020).

27 Kallenborn-Gerhardt, W., Schroder, K., Geisslinger, G. \& Schmidtko, A. NOXious signaling in pain processing. Pharmacol Ther 137, 309-317 (2013).

28 Fox, R. J. et al. BG-12 (dimethyl fumarate): a review of mechanism of action, efficacy, and safety. Curr Med Res Opin 30, 251-262 (2014).

29 Valko, M. et al. Free radicals and antioxidants in normal physiological functions and human disease. Int J Biochem Cell Biol 39, 44-84 (2007).

30 Price, T. J. et al. Transition to chronic pain: opportunities for novel therapeutics. Nat Rev Neurosci 19, 383-384 (2018).

31 Laras, Y. et al. Synthesis of Quinoline Dicarboxylic Esters as Biocompatible Fluorescent Tags. J Org Chem 77, 8294-8302 (2012).

32 Mast, C. A. et al. Efficient and versatile stereoselective synthesis of cryptophycins. Chem-Eur J 11, 4667-4677 (2005).

33 Kim, J. I. \& Ryu, C. M. Palladium Catalyzed Carbonylative Vinylation of Aryl Halides with Olefins and Carbon-Monoxide. B Kor Chem Soc 8, 246-250 (1987).

34 Shields, S. D., Eckert, W. A. \& Basbaum, A. I. Spared nerve injury model of neuropathic pain in the mouse: A behavioral and anatomic analysis. Journal of Pain 4, 465-470 (2003).

35 Chaplan, S. R., Bach, F. W., Pogrel, J. W., Chung, J. M. \& Yaksh, T. L. Quantitative assessment of tactile allodynia in the rat paw. J Neurosci Methods 53, 55-63 (1994).

36 Duan, B. et al. Identification of spinal circuits transmitting and gating mechanical pain. Cell 159, 1417-1432 (2014).

37 Blackburn-Munro, G. \& Jensen, B. S. The anticonvulsant retigabine attenuates nociceptive behaviours in rat models of persistent and neuropathic pain. Eur $J$ Pharmacol 460, 109-116 (2003).

38 Bouet, V. et al. The adhesive removal test: a sensitive method to assess sensorimotor deficits in mice. Nat Protoc 4, 1560-1564 (2009). 


\section{FIGURES}

Figure 1. In vitro characterization of 1,2-dicarbonyl compounds. (a) MMF generating 1,2-dicarbonyl compounds and their reaction with aqueous hydrogen peroxide and peroxynitrite. (b-e) NRF2/antioxidant response element luciferase reporter HEK293 cells were treated with media, $\mathrm{H}_{2} \mathrm{O}_{2}(10 \mu \mathrm{M})$, or ONOO' $(20 \mu \mathrm{M})$. AREluciferase activity was quantified following treatment with concentration ranges of MMF or 1,2-dicarbonyl compounds (b) $\mathbf{1 a}$, (c) 1b, or (d) 1c; not significant (n.s.), ${ }^{* *} P<0.01$, ${ }^{* * *} P<0.001$. (e) Expression of Nrf2 target genes (Sod1, Hmox1, and Gclm) was quantified after treatment with 1c or MMF $(20 \mu \mathrm{M})$; relative to media: ${ }^{* \star} P<0.01,{ }^{* \star \star} P<$ 0.001 ; relative to vehicle: ${ }^{\# \#} P<0.01,{ }^{\# \#} P<0.001$. (f) Chemical structure of 1,2dicarbonyl 1c.

Figure 2. In vivo assessment of 1c efficacy and site-selectivity. (a) Schematic of spared nerve injury and tissues of interest. $(\mathbf{b}, \mathbf{c})$ Once neuropathic pain was established on day 7 after SNI, male $(n=6)$ and female $(n=6)$ mice were treated with oral

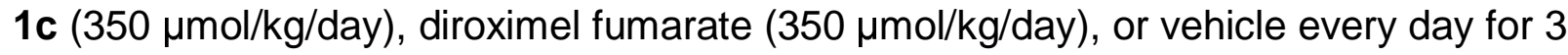
days (gray box). (b) Mechanical allodynia and (c) dynamic allodynia were assessed. Relative to vehicle: ${ }^{*} P<0.05,{ }^{* *} P<0.01,{ }^{* \star} P<0.001 ; 1 \mathrm{c}$ vs. diroximel fumarate: ${ }^{\# \#} P$ $<0.01$, \#\# $P<0.001$. (d) Seven days after SNI, male $(n=3)$ and female $(n=3)$ mice were treated with oral $1 \mathrm{c}(350 \mu \mathrm{mol} / \mathrm{kg} /$ day $)$ or subcutaneous morphine (3 $\mathrm{mg} / \mathrm{kg}$, b.i.d.). Relative to 1c Day 0 (D0, prior to surgery): ${ }^{* *} P<0.01,{ }^{* * *} P<0.001$; relative to morphine D0: ${ }^{\#} P<0.05,{ }^{\# \#} P<0.001$. (e) Male $(\mathrm{n}=3)$ and female $(\mathrm{n}=3)$ mice were treated with oral 1c $(350 \mu \mathrm{mol} / \mathrm{kg} /$ day $)$, diroximel fumarate (350 $\mu \mathrm{mol} / \mathrm{kg} /$ day) or vehicle every day for 7 
days, beginning 7 days after SNI or sham surgery. Presence of ongoing pain was interpreted as an increase in time spent in retigabine-paired chamber (conditioning stimulus) after 7 days of treatment, relative to baseline. Relative to sham-vehicle: ${ }^{\dagger \dagger} P$ $<0.01$; relative to SNI-vehicle: ${ }^{*} P<0.05,{ }^{* \star *} P<0.001$. (f) Male and female $N f e 2 / 2^{/-}$and wildtype control mice ( $n=3 / \mathrm{sex} /$ group) were treated with oral $1 \mathrm{c}(350 \mu \mathrm{mol} / \mathrm{kg} / \mathrm{day})$, beginning 7 days after SNI, and mechanical allodynia assessed. Relative to wildtype controls: ${ }^{* * *} P<0.001$. (g) $L 4 / 5$ DRG from 3 mice were pooled after 3 days of treatment, and nuclear extracts were probed for Nrf2 ( $n=2$ males, $n=2$ females). Relative to ipsilateral vehicle: ${ }^{* *} P<0.001$; relative to contralateral vehicle and 1c: ${ }^{t \dagger} P<0.001$. (h) Antioxidant gene expression L4/5 DRG was determined after 3 days of treatment $(n=3$ males, $\mathrm{n}=3$ females). Relative to ipsilateral vehicle: ${ }^{*} P<0.05,{ }^{* \star} P<0.01$; relative to contralateral vehicle: ${ }^{\#} P<0.05$; relative to contralateral 1c: ${ }^{\dagger} P<0.05$; ${ }^{\dagger \dagger} P<0.01,{ }^{\mathrm{tt}} P$ $<0.001$. (i) Naive male $(n=3)$ and female $(n=3)$ mice were orally treated with $1 c$ (350 $\mu \mathrm{mol} / \mathrm{kg})$, diroximel fumarate $(350 \mu \mathrm{mol} / \mathrm{kg})$, or vehicle daily for 10 days. Blood was collected by cardiac puncture and leukocytes were manually counted. Relative to vehicle: ${ }^{\star} P<0.05$. Individual replicates are presented in spaghetti plots or grey dots. 
a<smiles>[R]C(=O)C(=O)/C=C/C(=O)OC</smiles>
1a: $R=P h$
1b: $\mathrm{R}=\mathrm{NHBn}$
1c: $\mathrm{R}=\mathrm{OMe}$
1d: $\mathrm{R}=\mathrm{OH}$

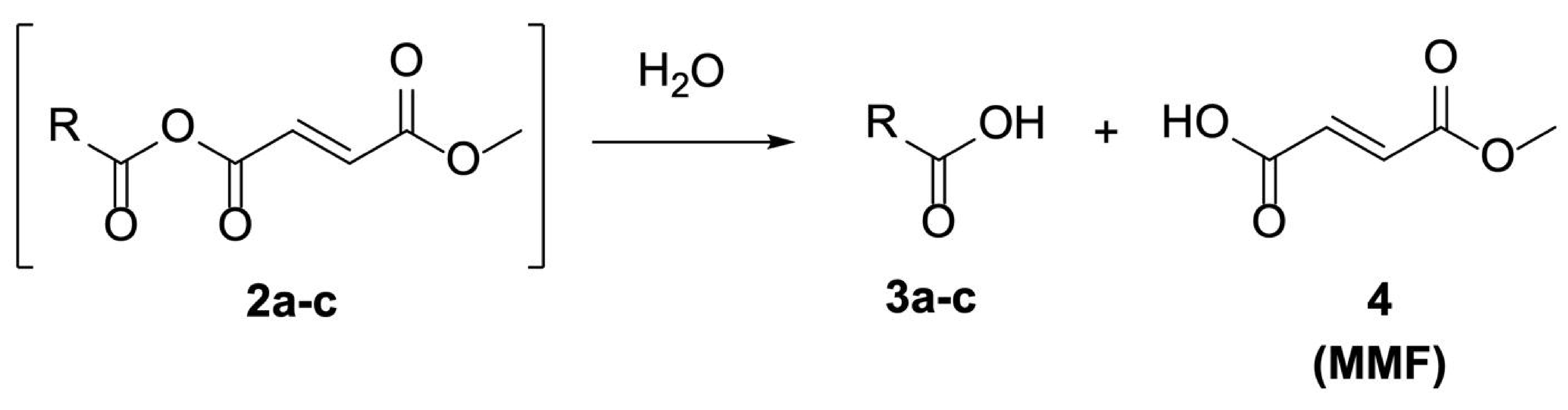

b

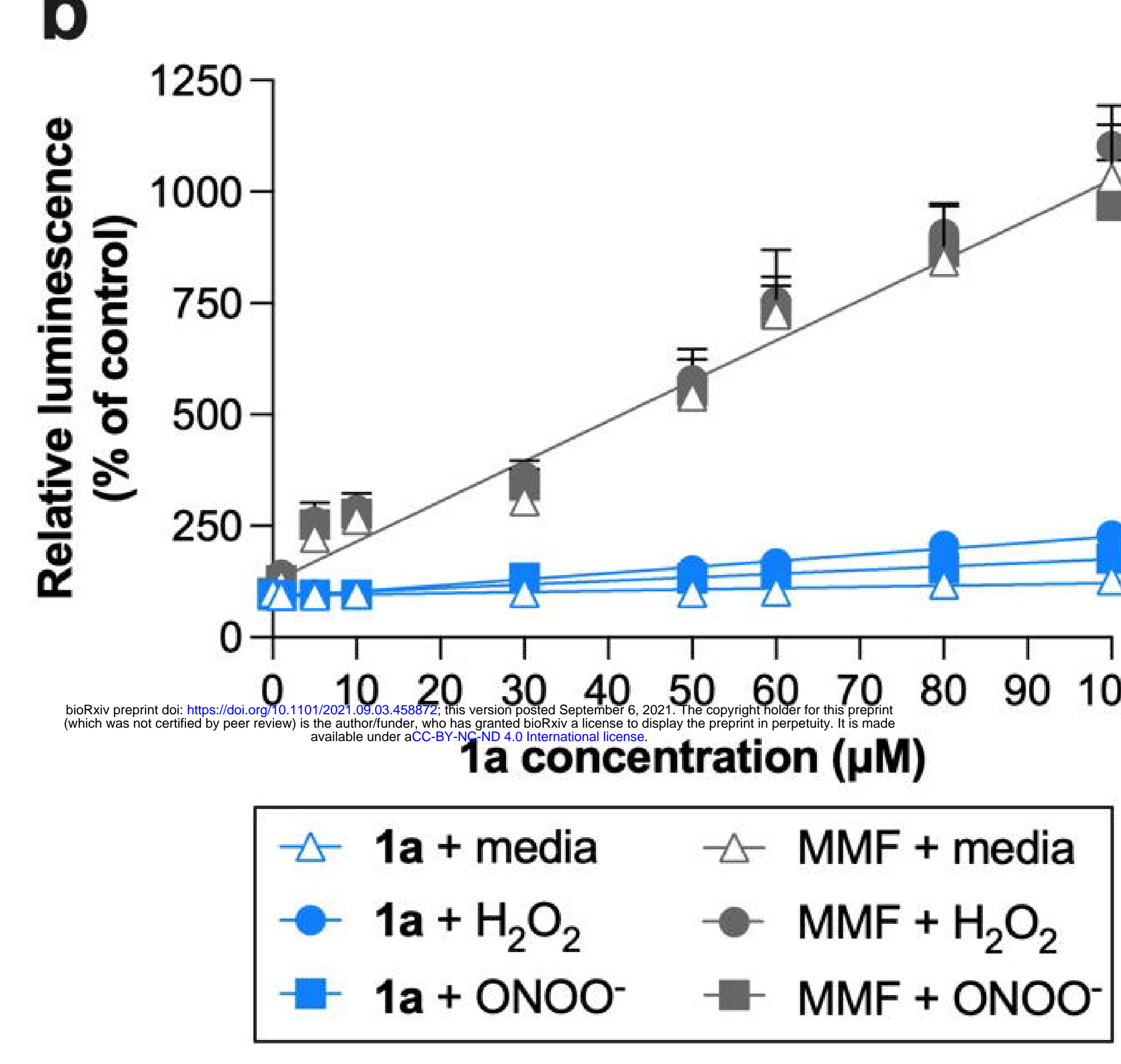

C
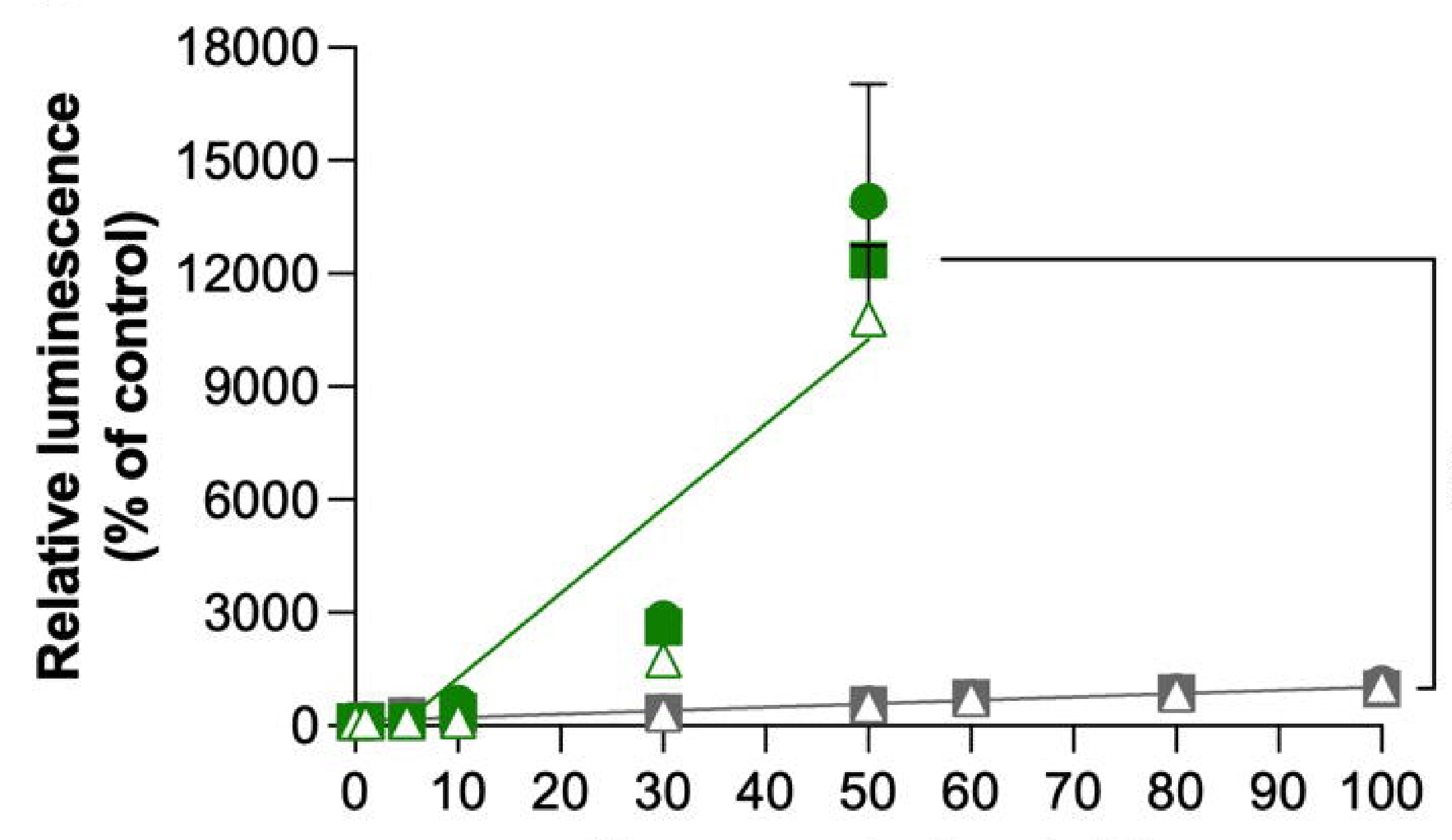

$1 \mathrm{~b}$ concentration $(\mu \mathrm{M})$

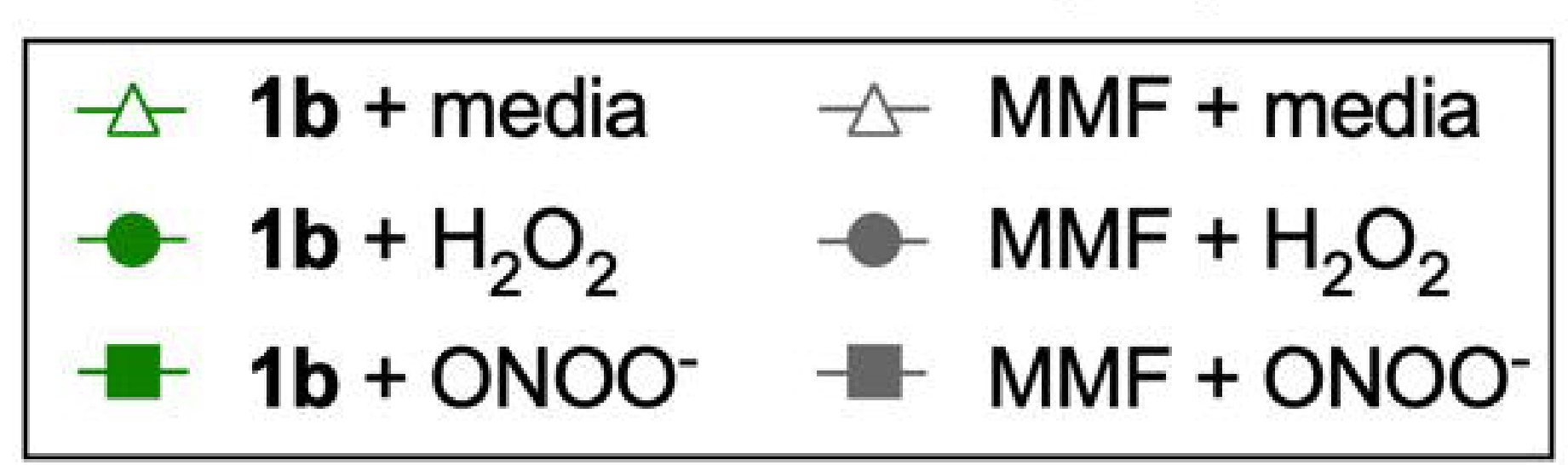

d

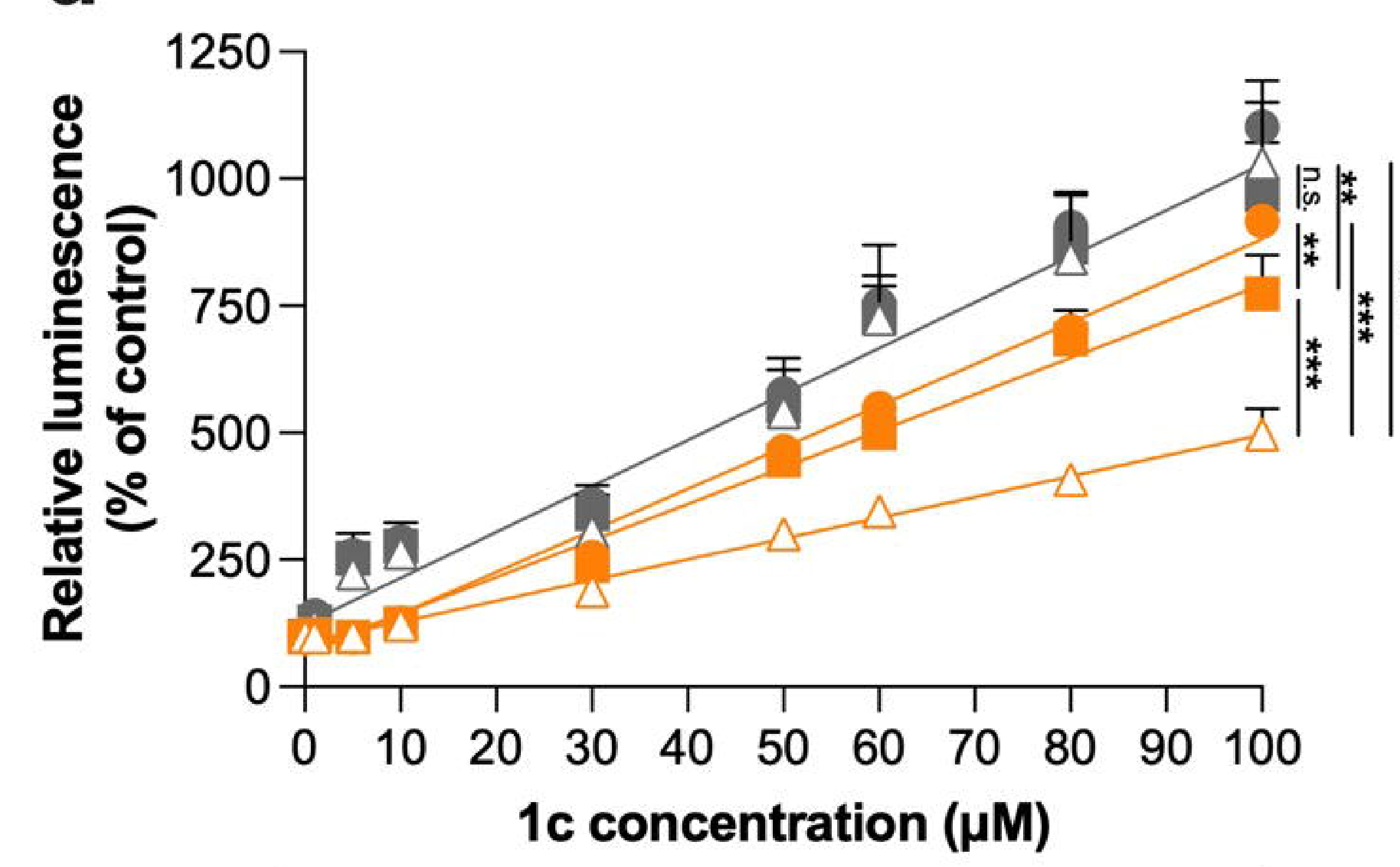

e

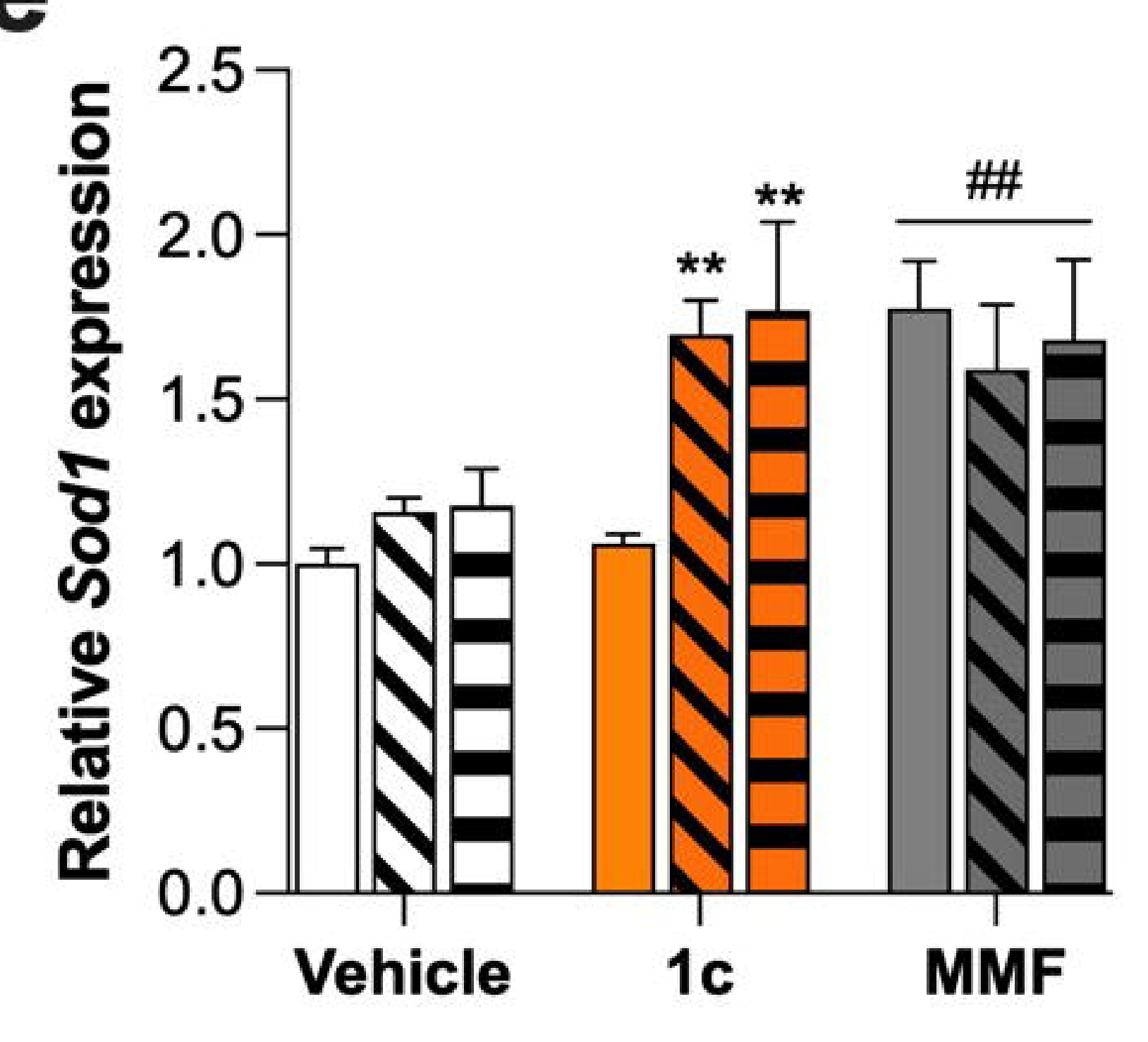

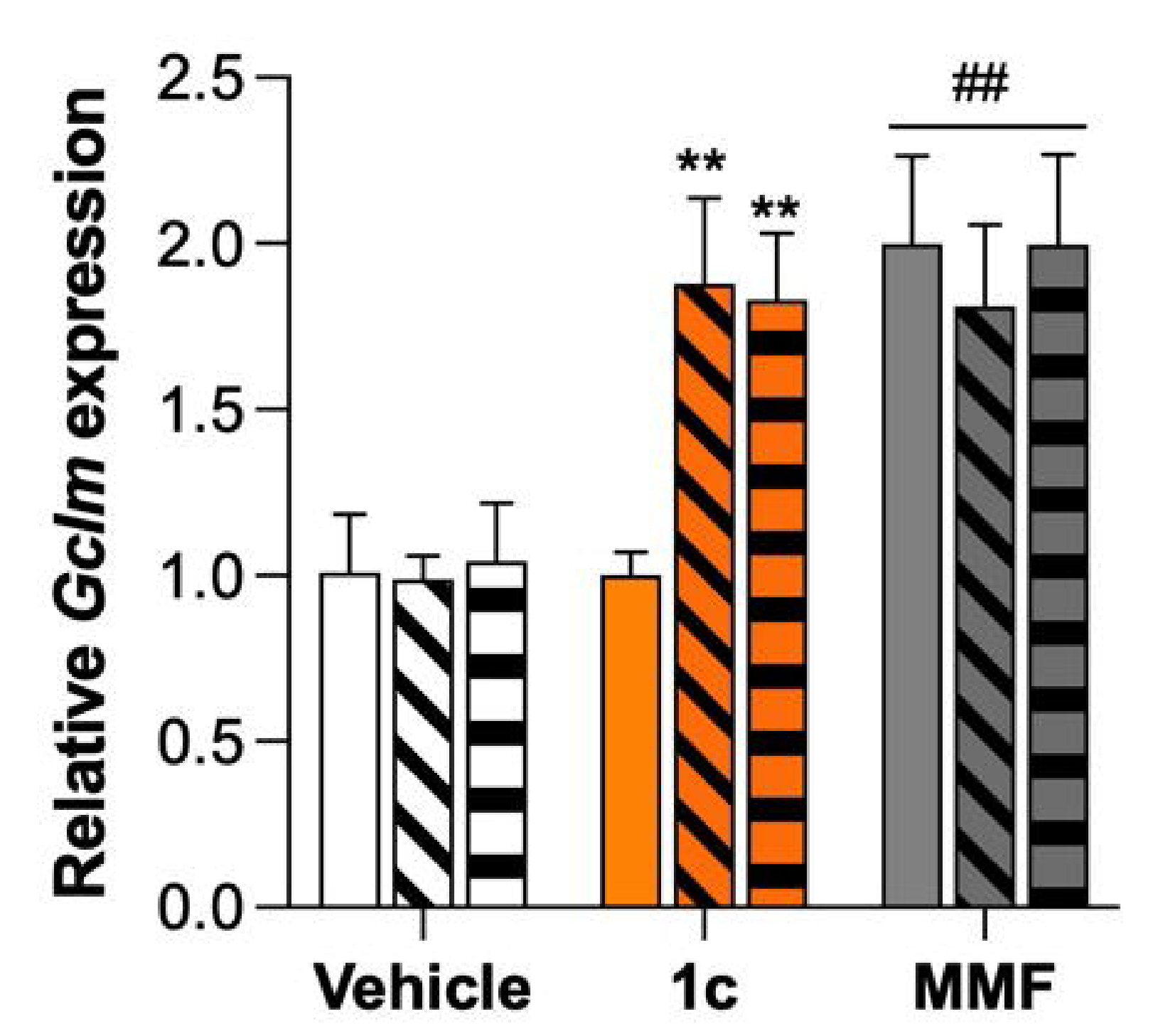

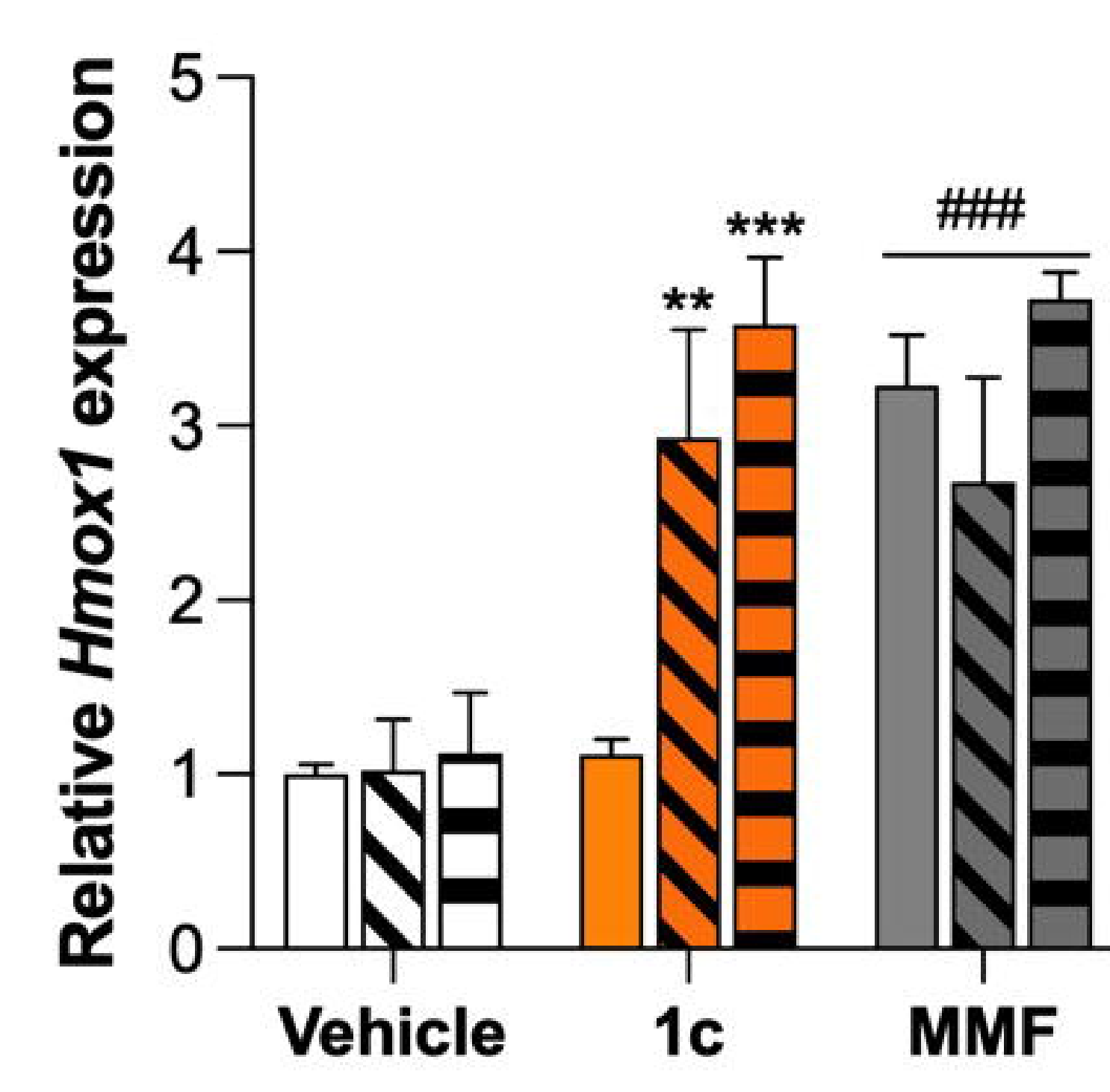

f<smiles>COC(=O)/C=C/C(=O)C(=O)OC</smiles>

1c 
a

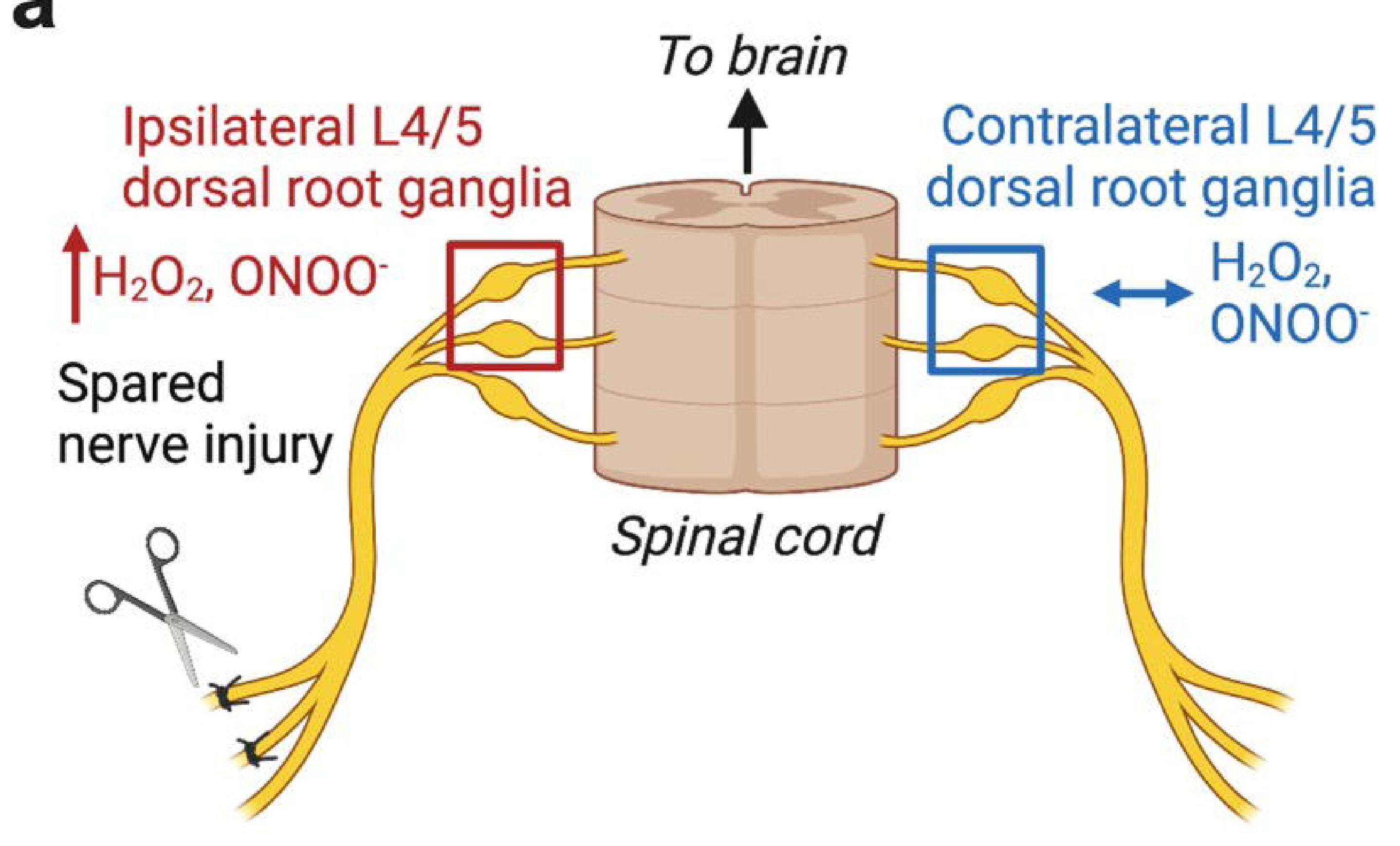

d

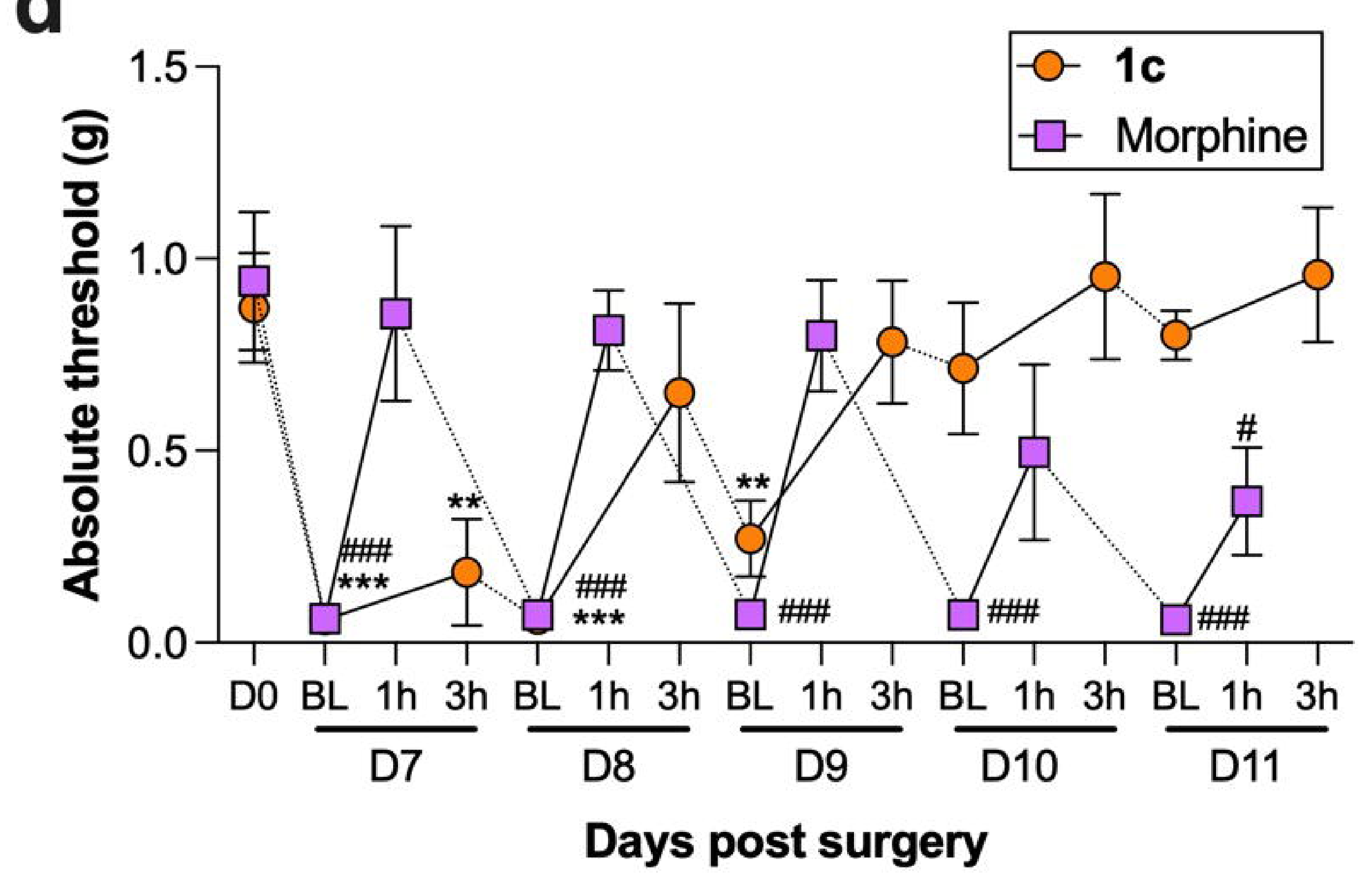

g
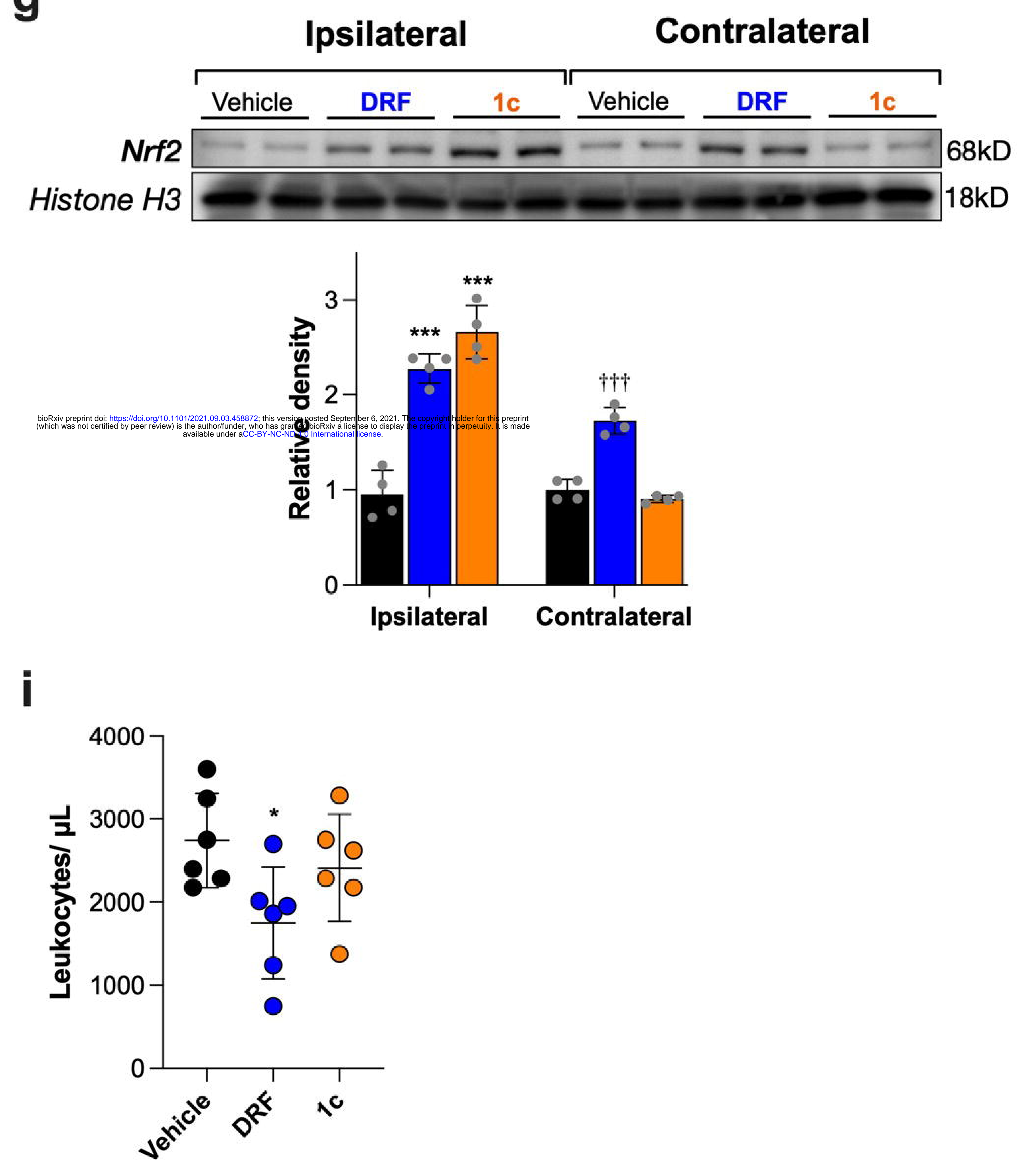

e b

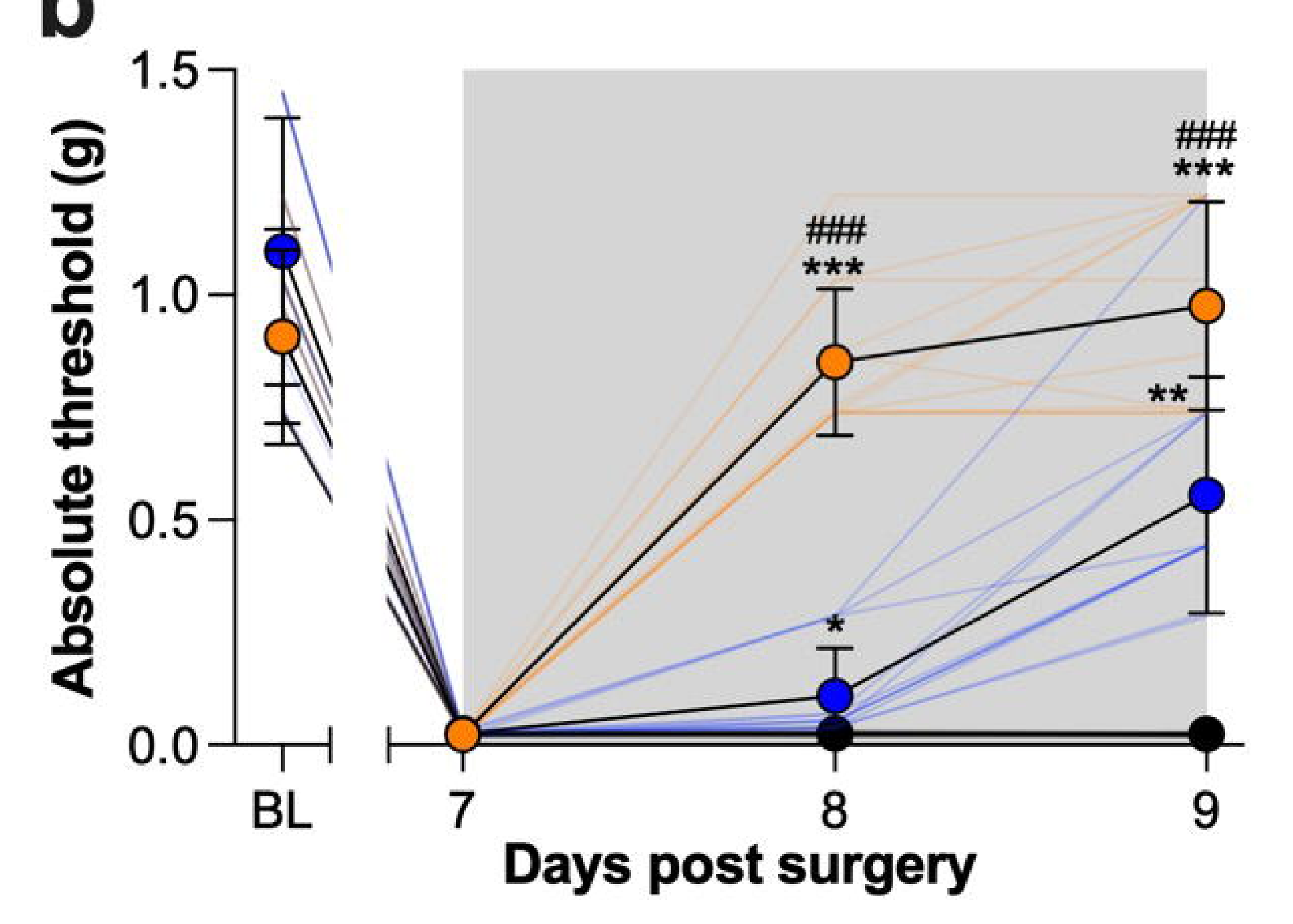

C

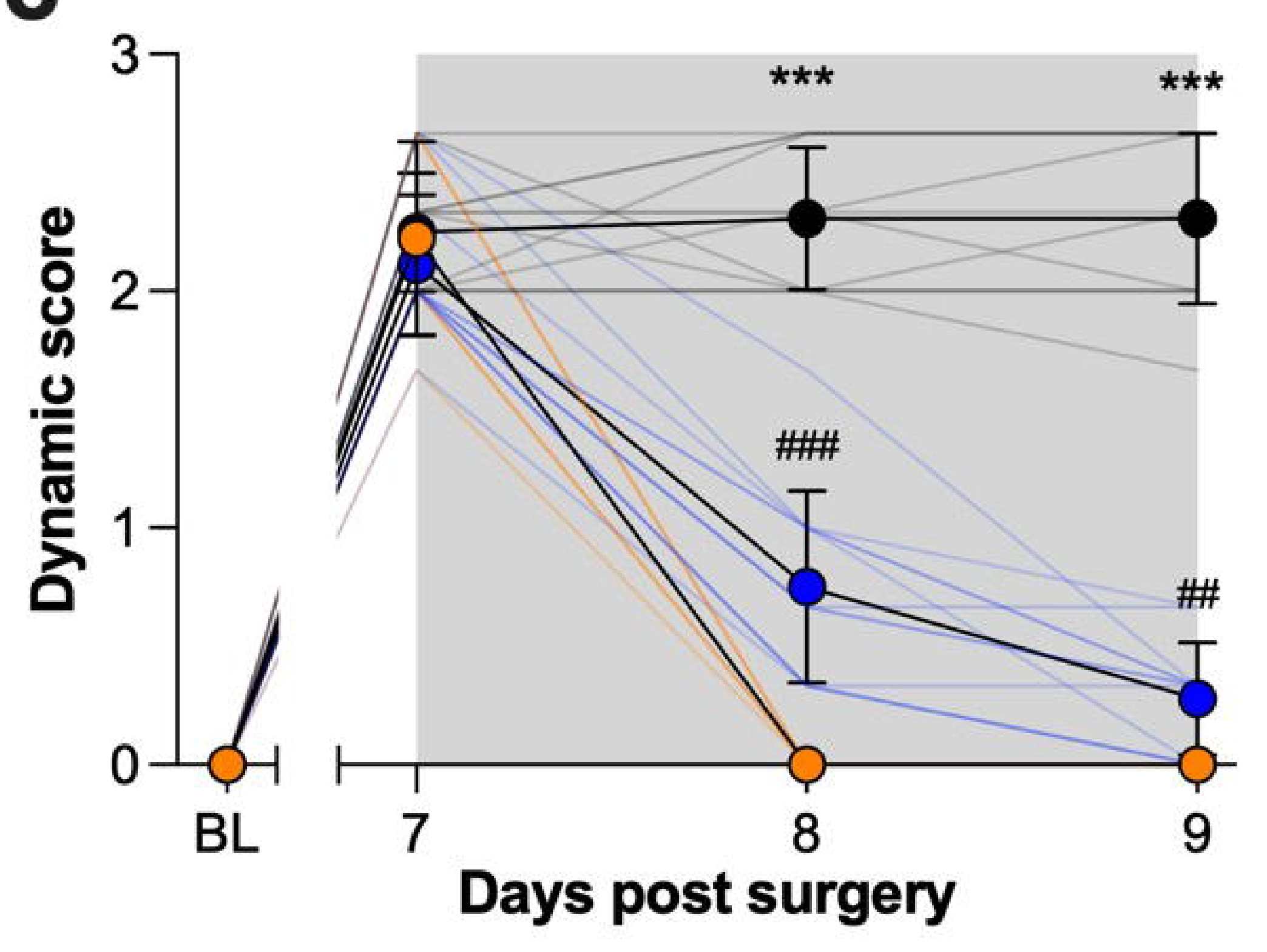

- Vehicle - Diroximel fumarate -o 1c

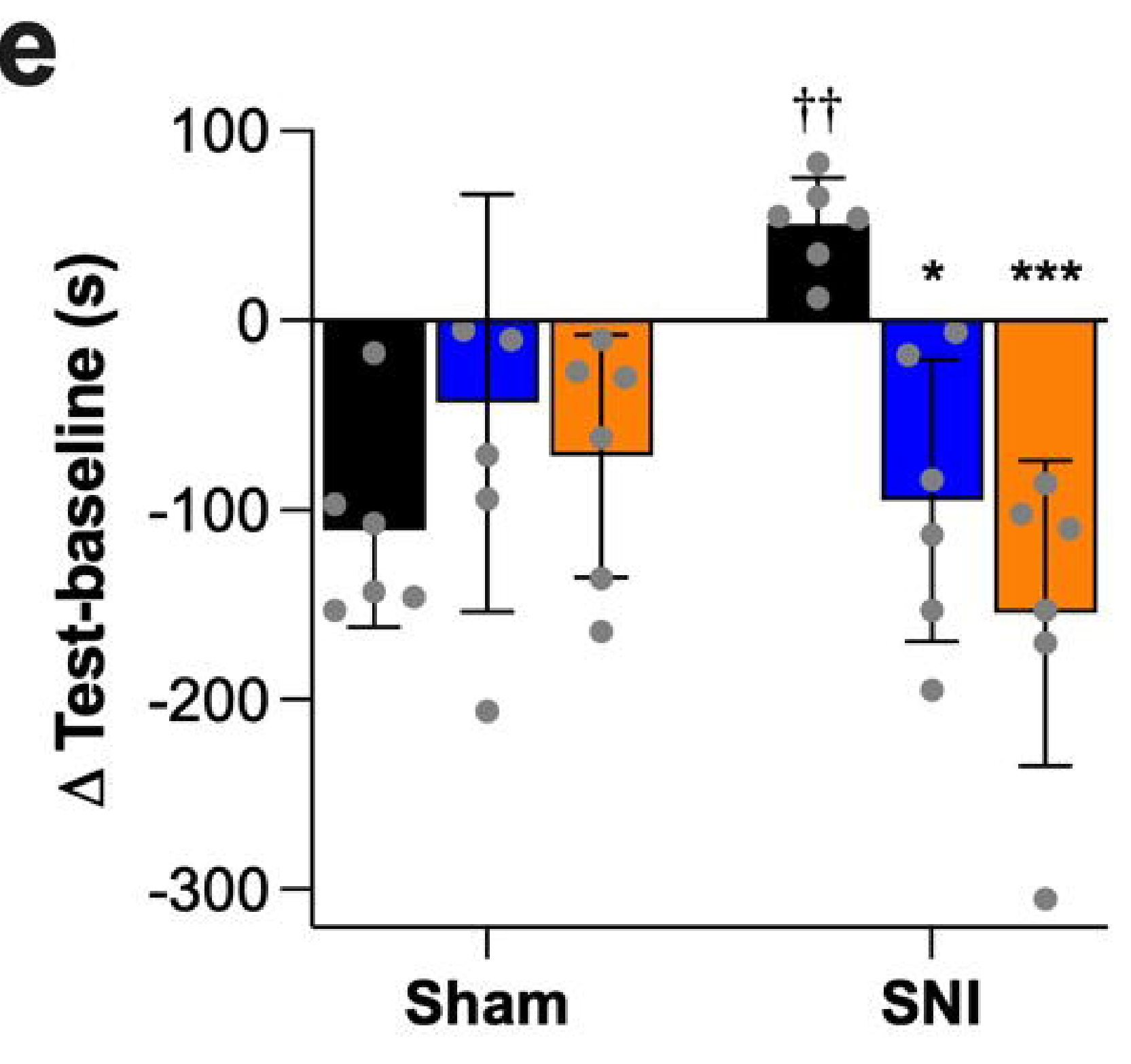

h
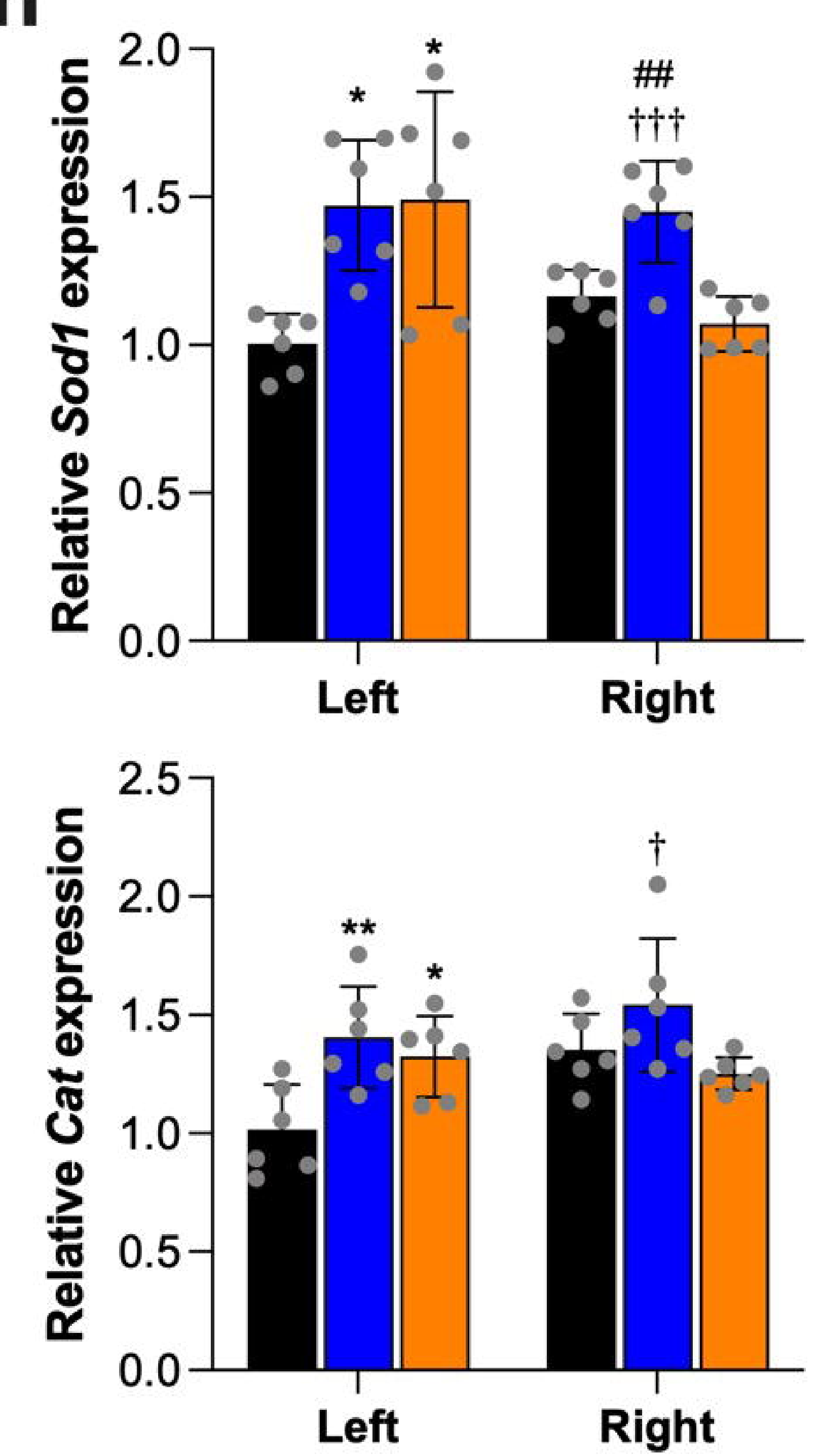

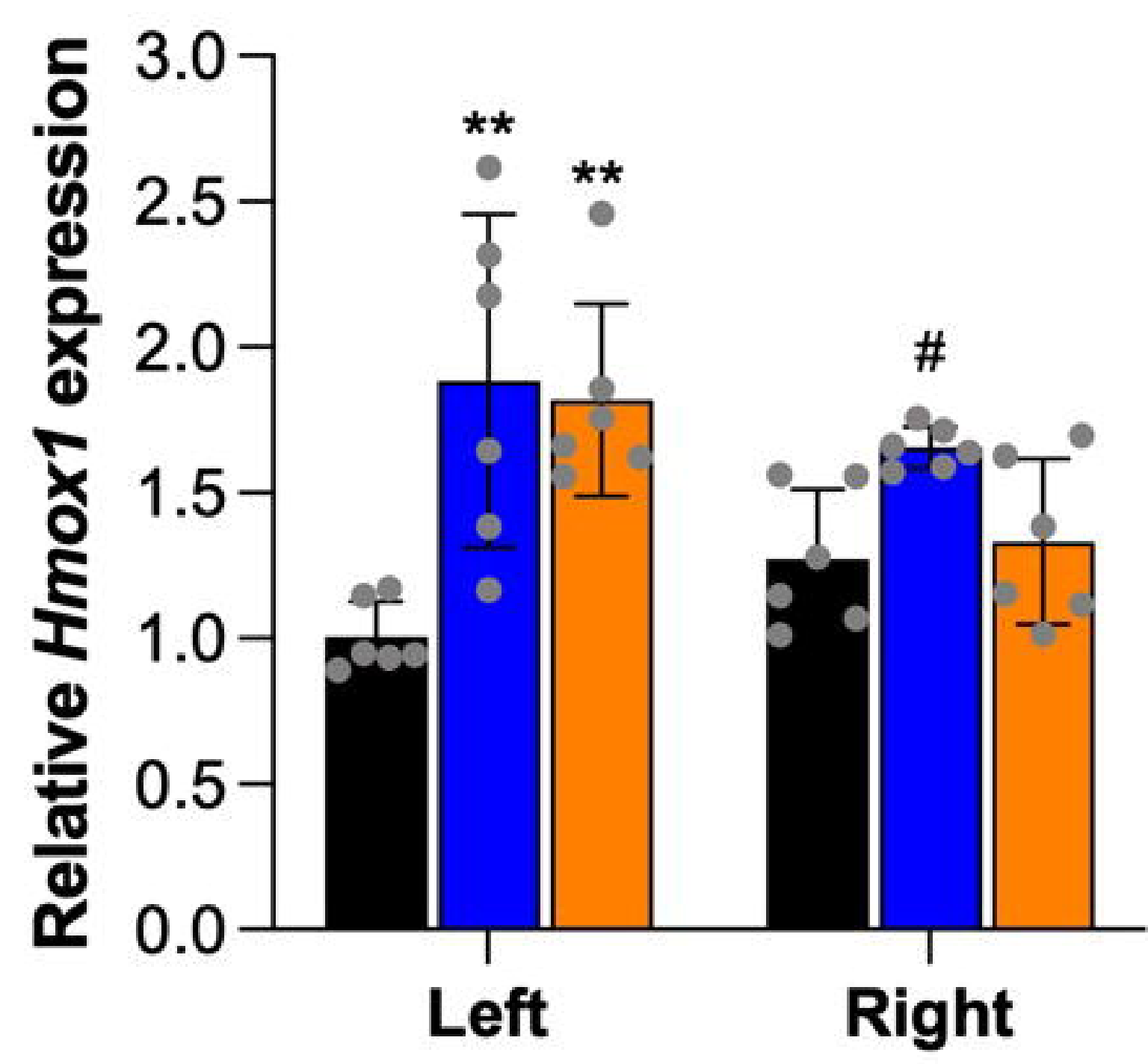

$f$
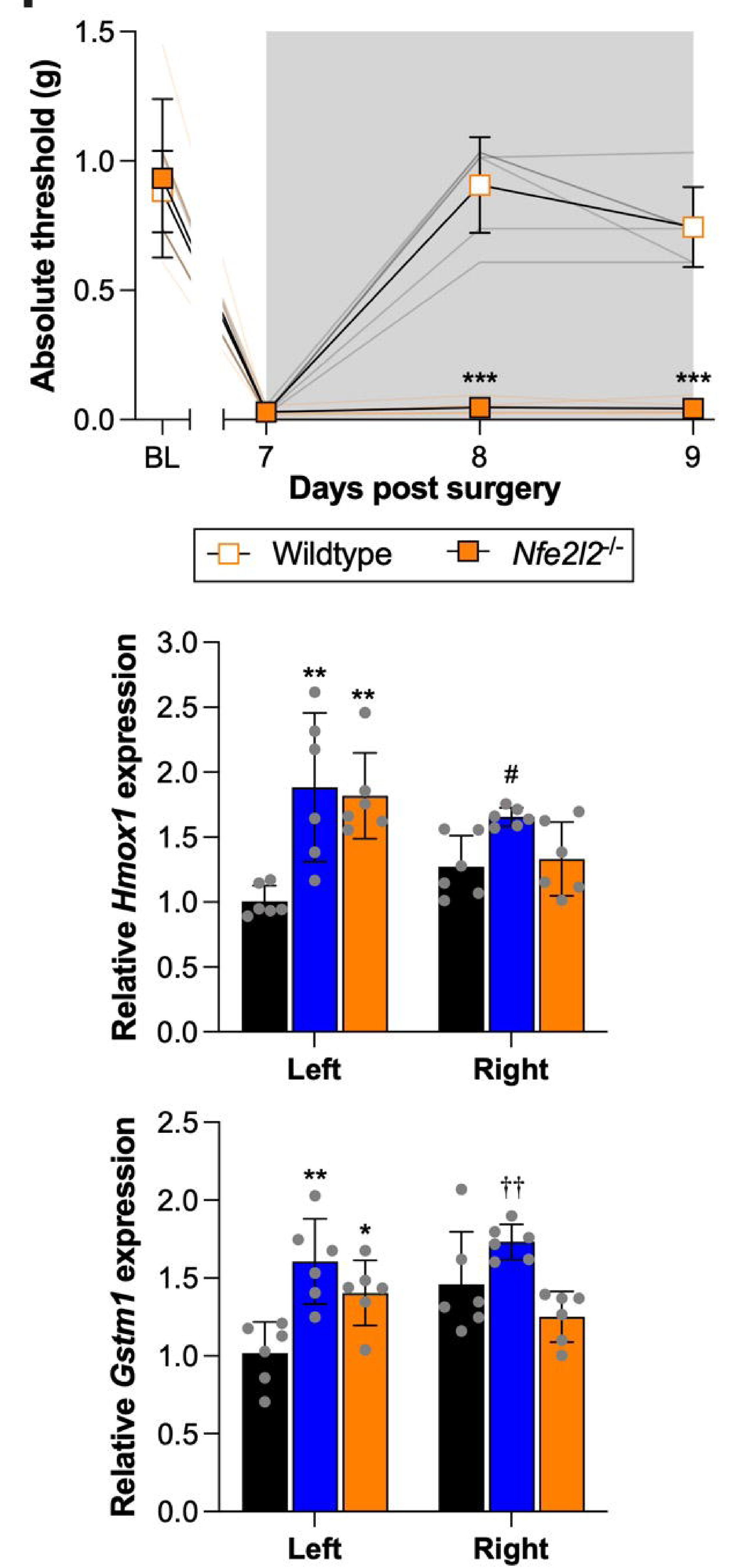
a

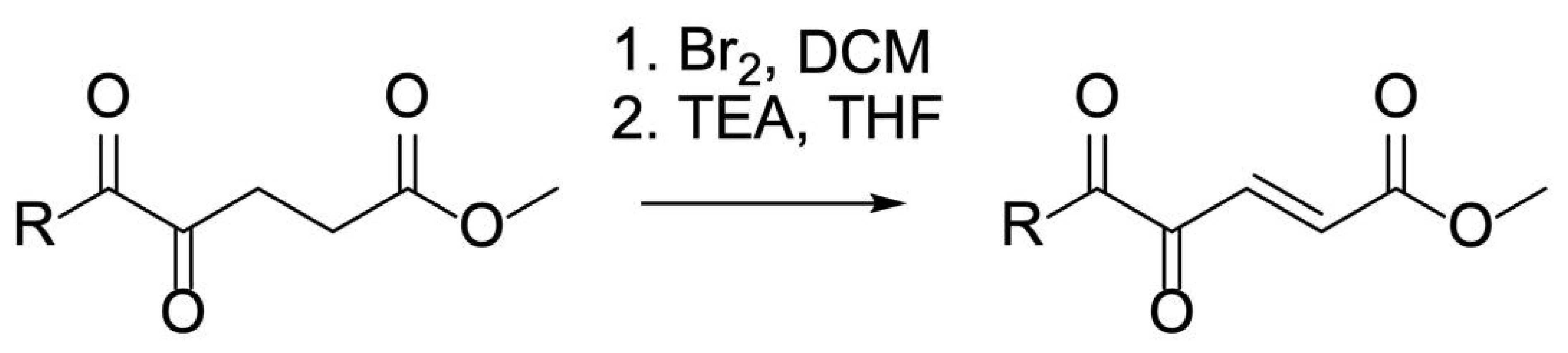

5a: $\mathrm{R}=\mathrm{NHBn}$

5b: $\mathrm{R}=\mathrm{OMe}$

b

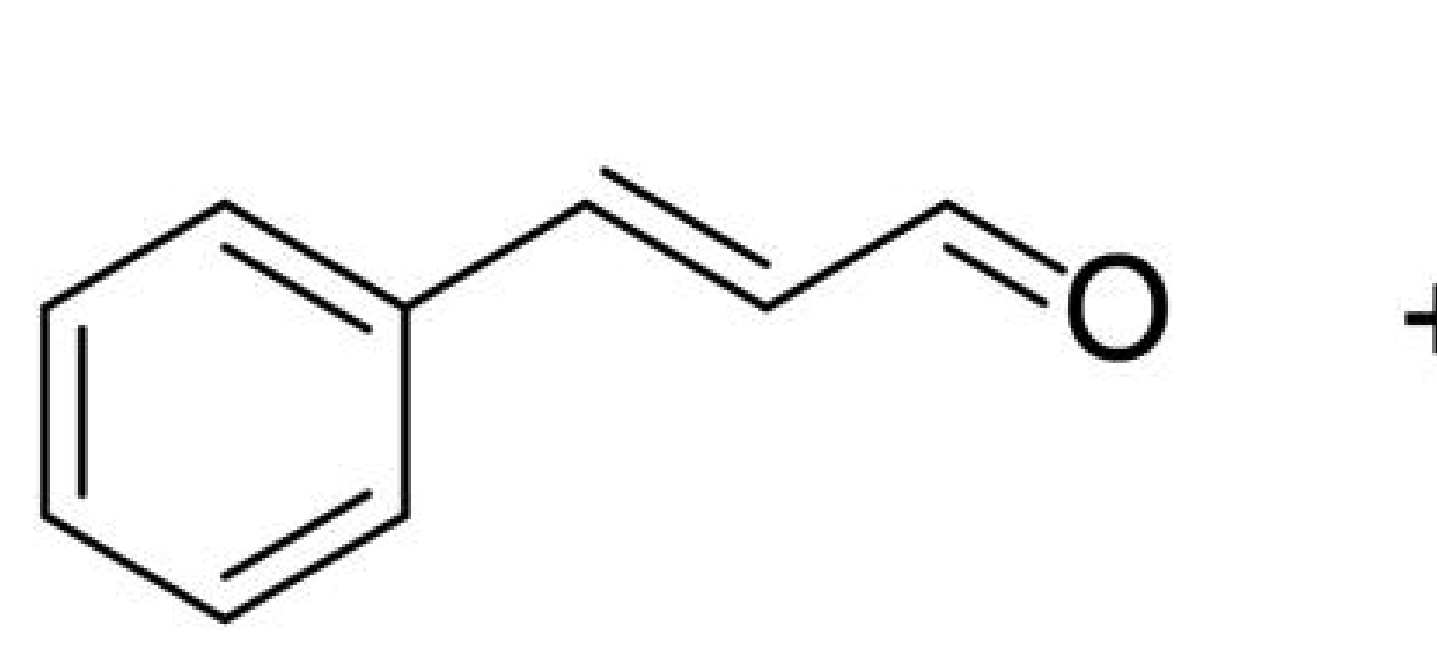

6<smiles>COC(=O)C=P</smiles>

7 1b: $\mathrm{R}=\mathrm{NHBn}$

1c: $\mathrm{R}=\mathrm{OMe}$ 


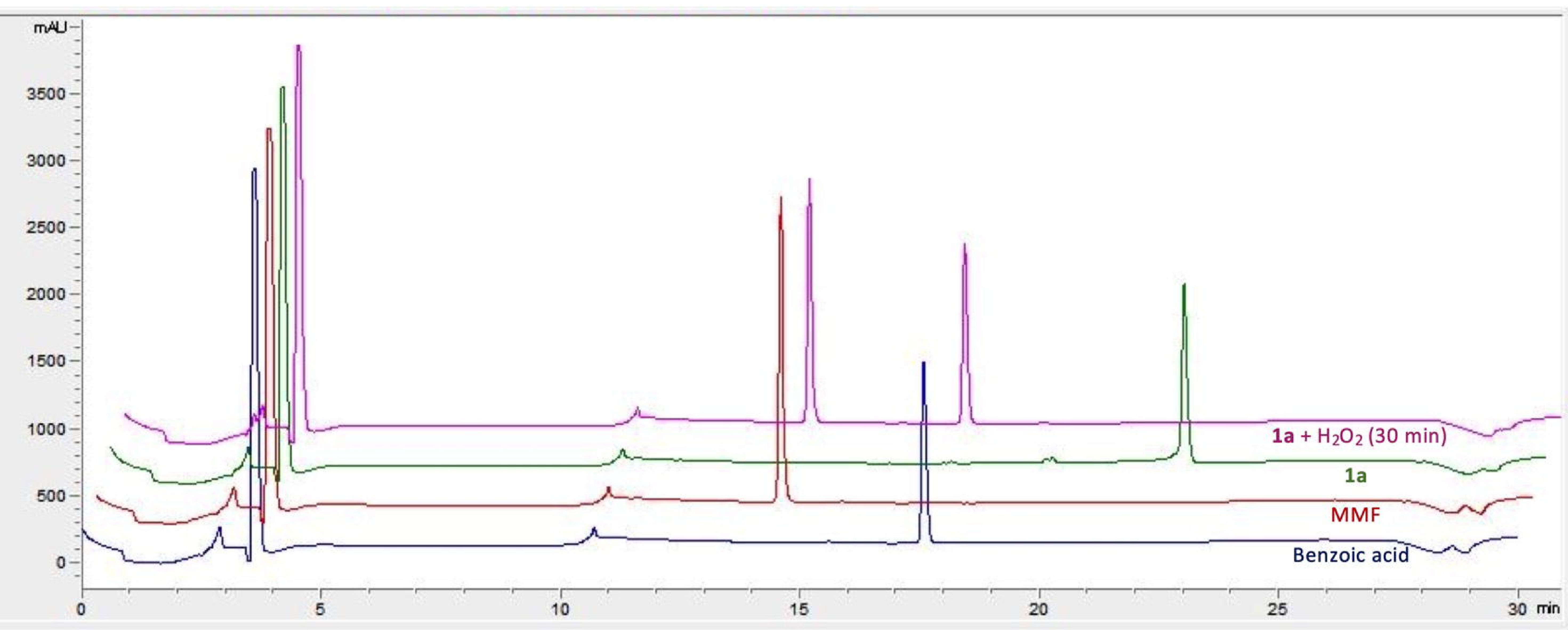

b

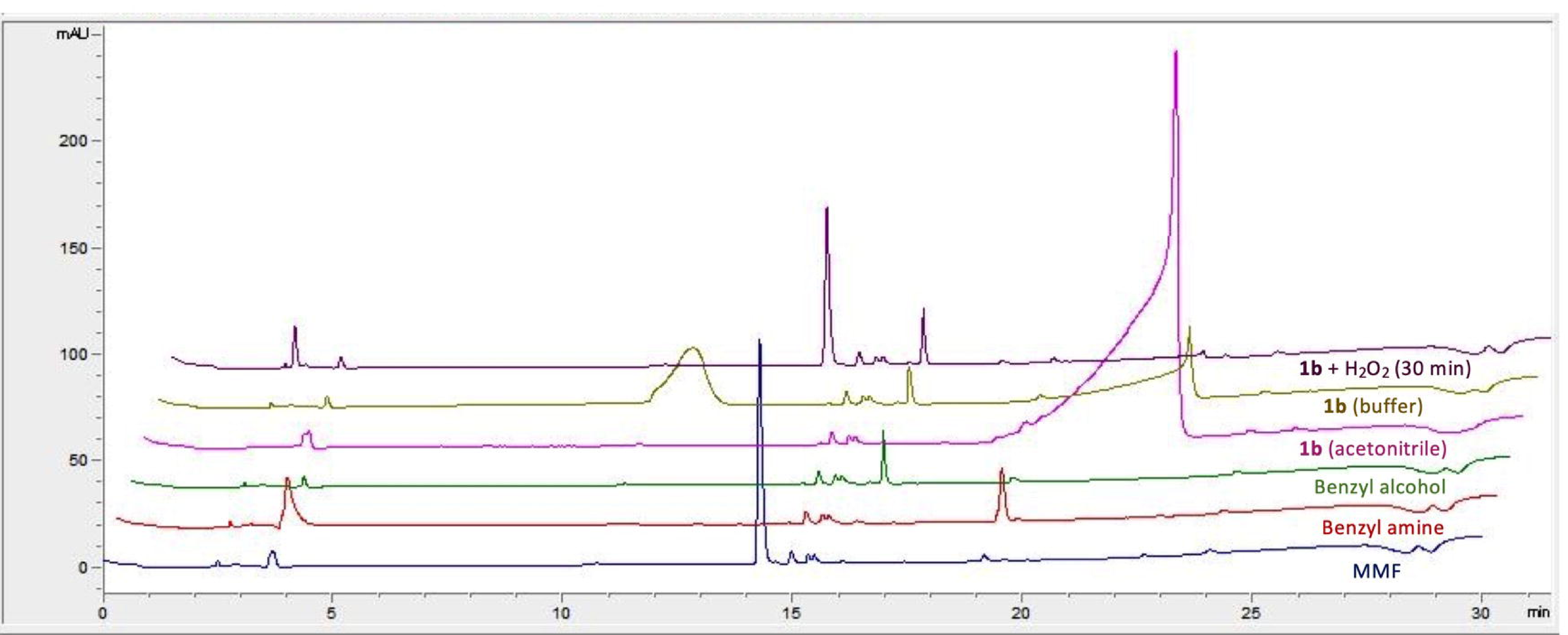

c

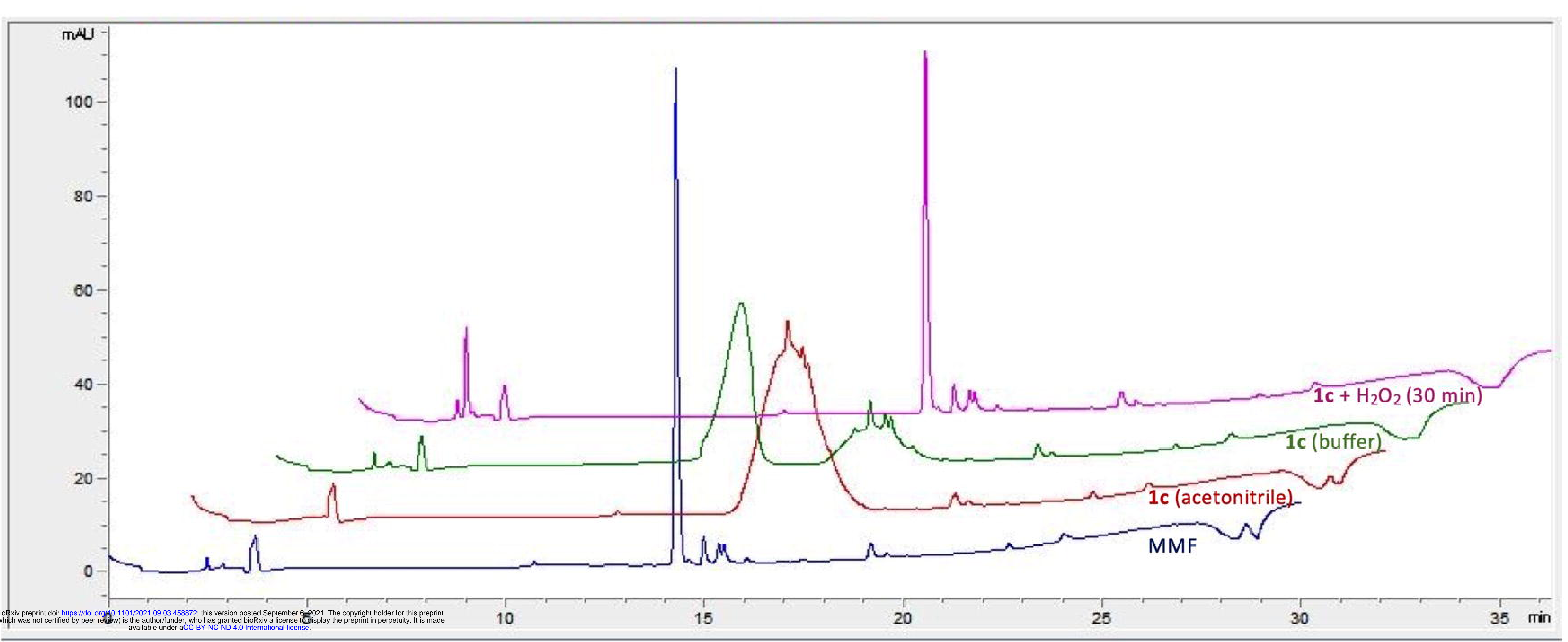

d

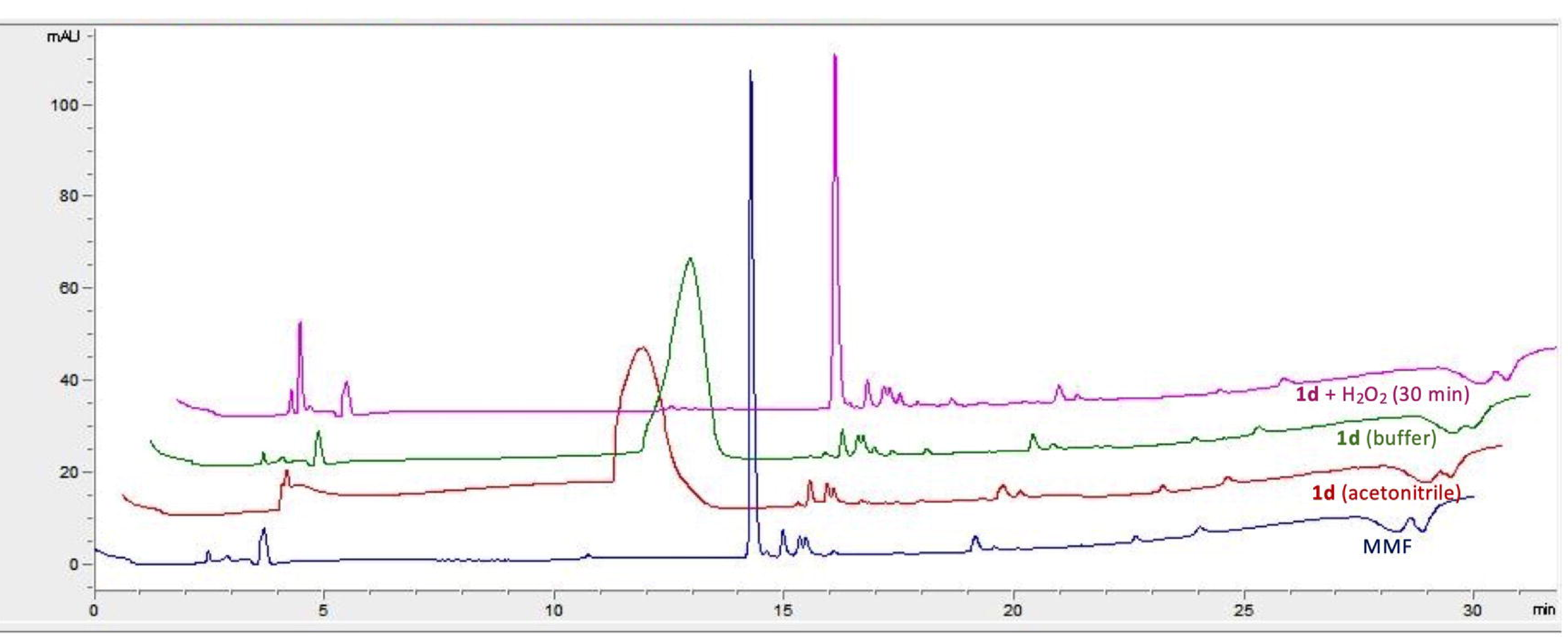




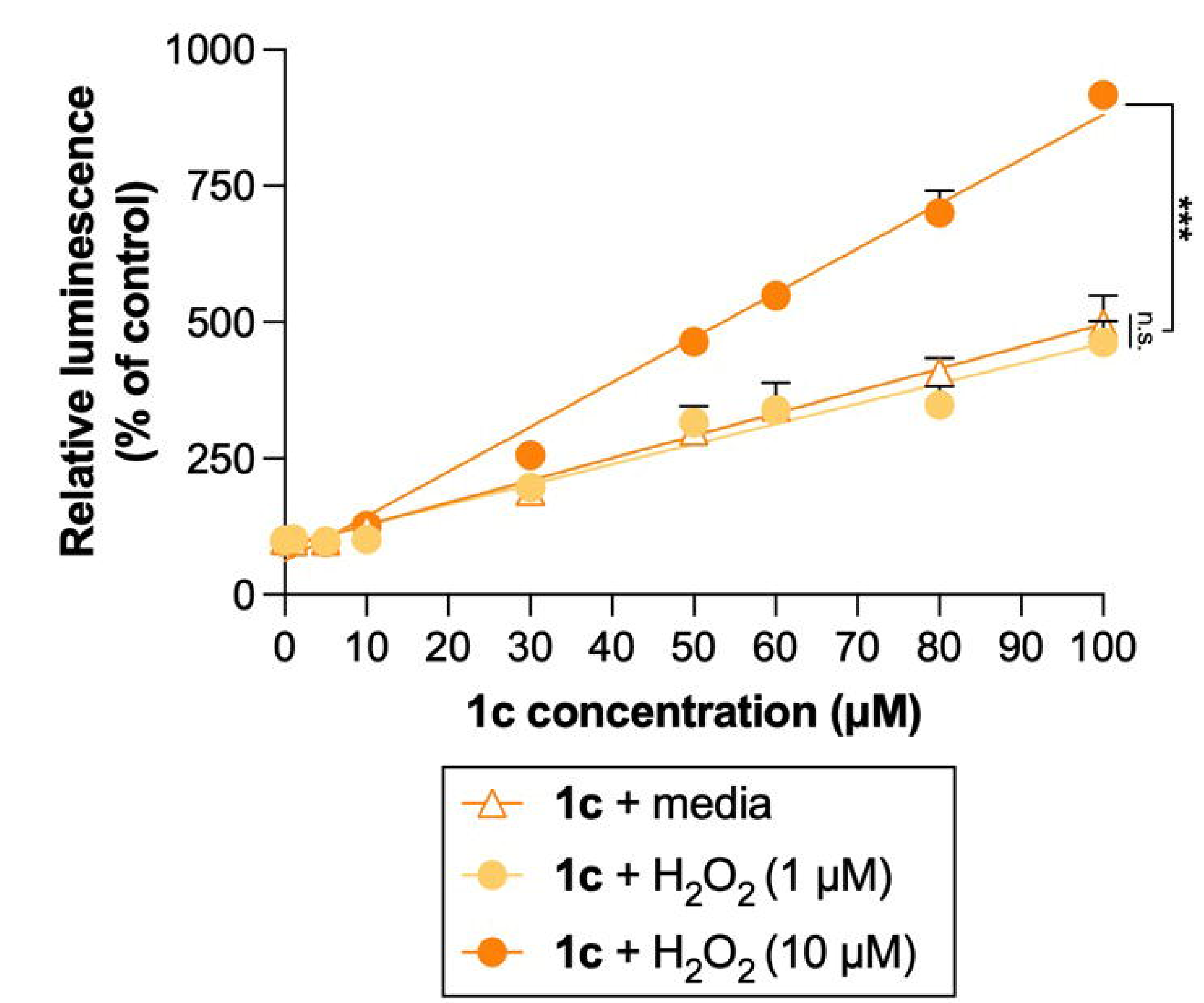

b $\quad$ c

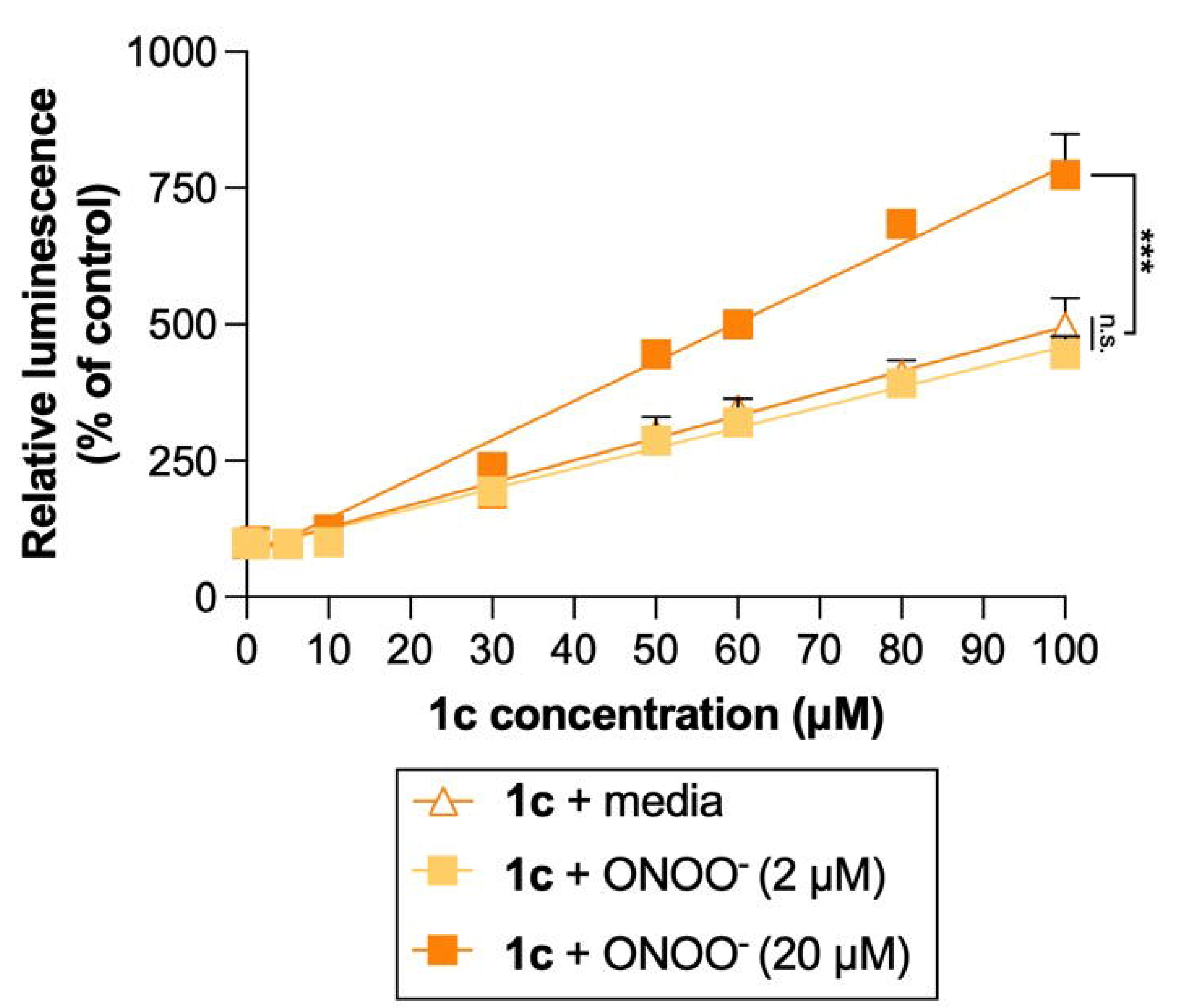

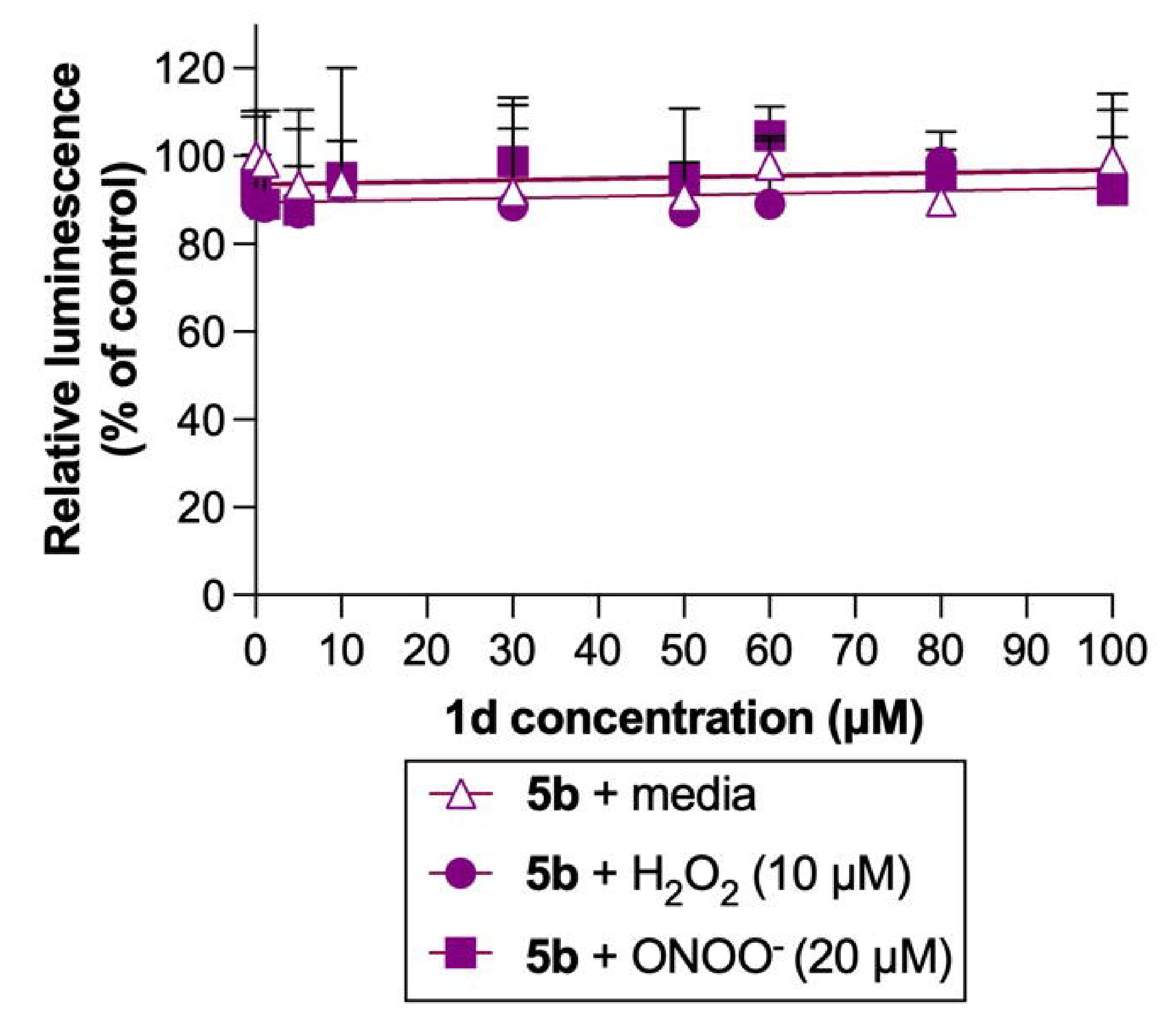


Sciatic nerve

Histone $\mathrm{H} 3$
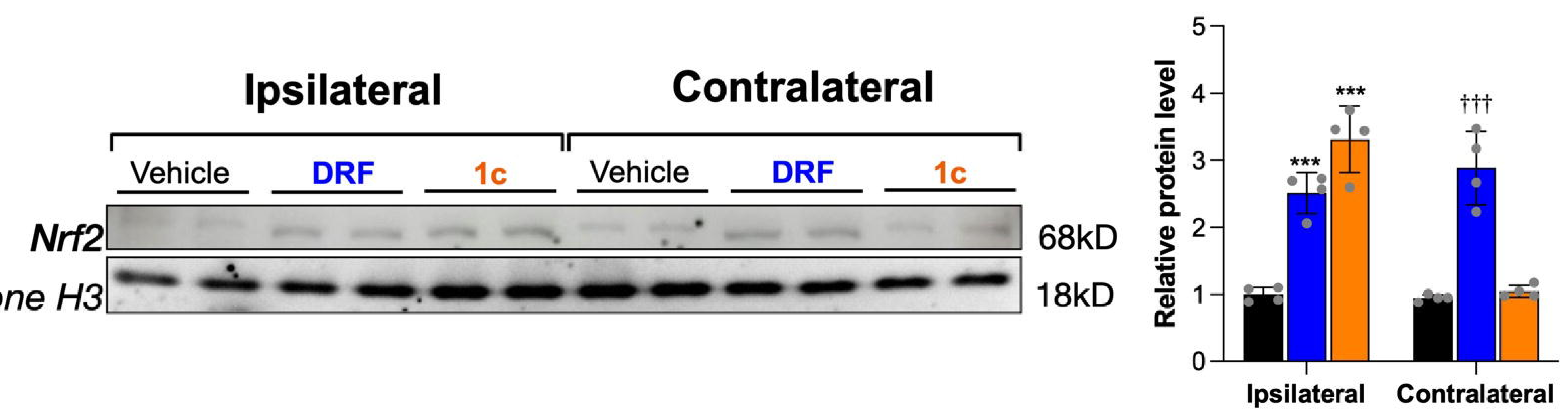

Liver

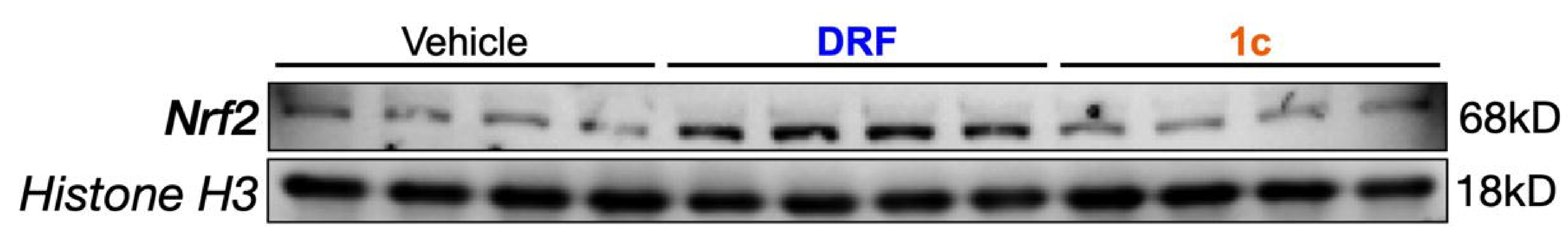

Kidney

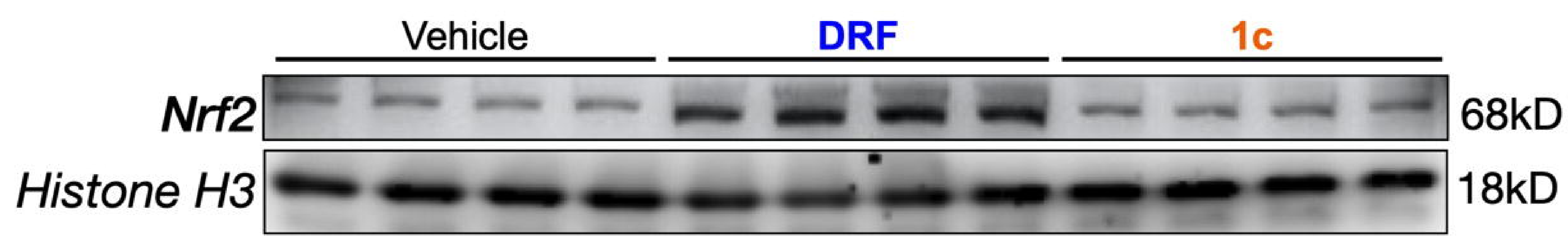

\section{Lung}

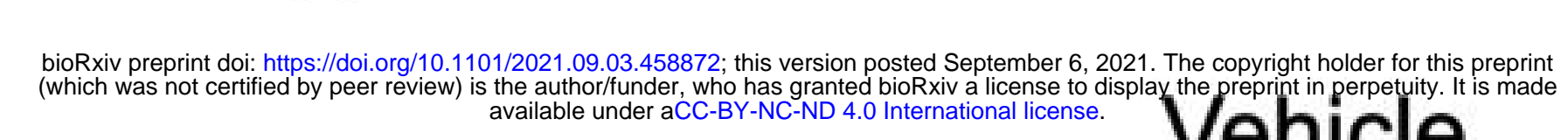

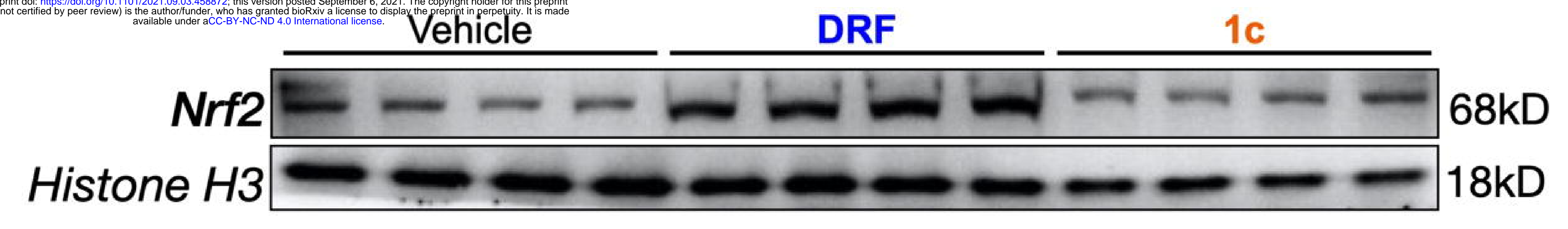

\section{DRG}

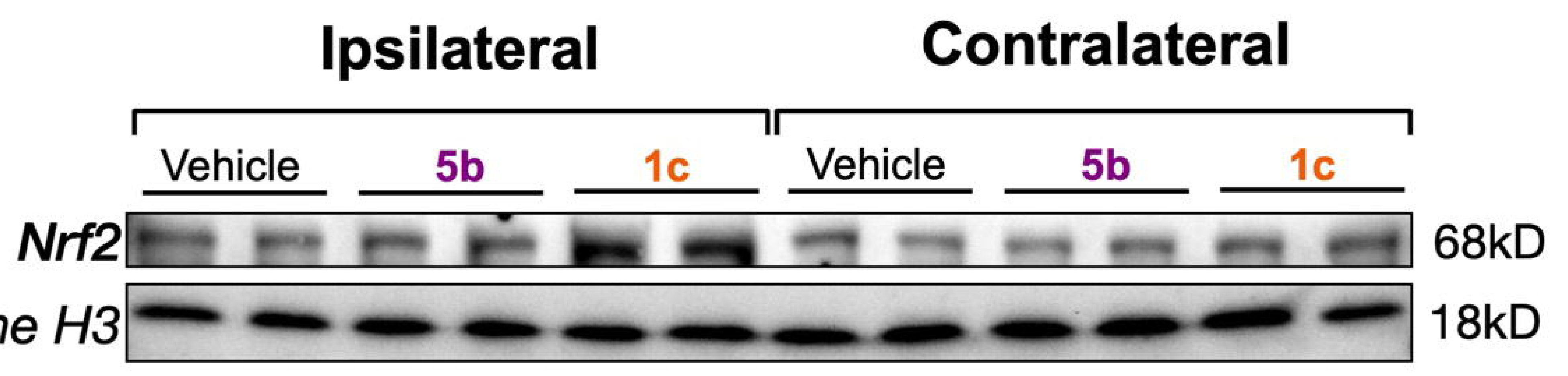

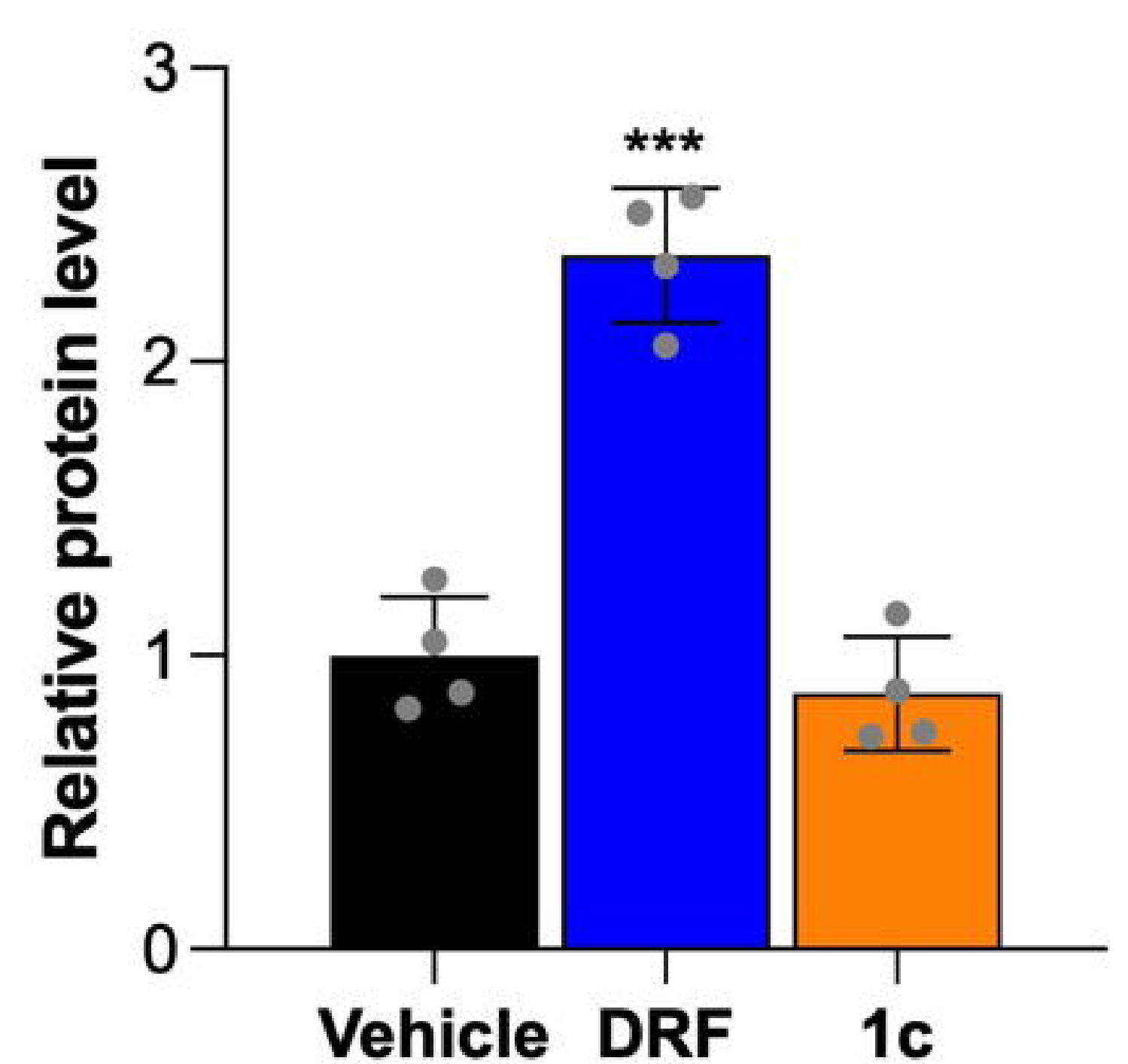
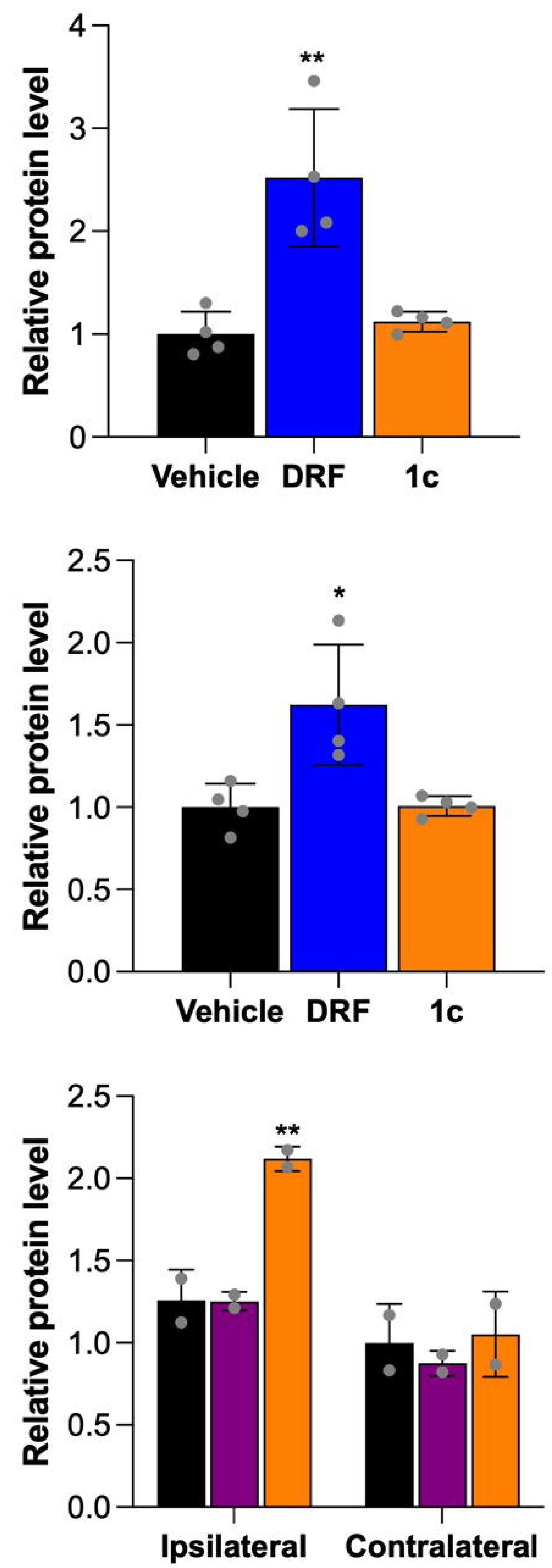


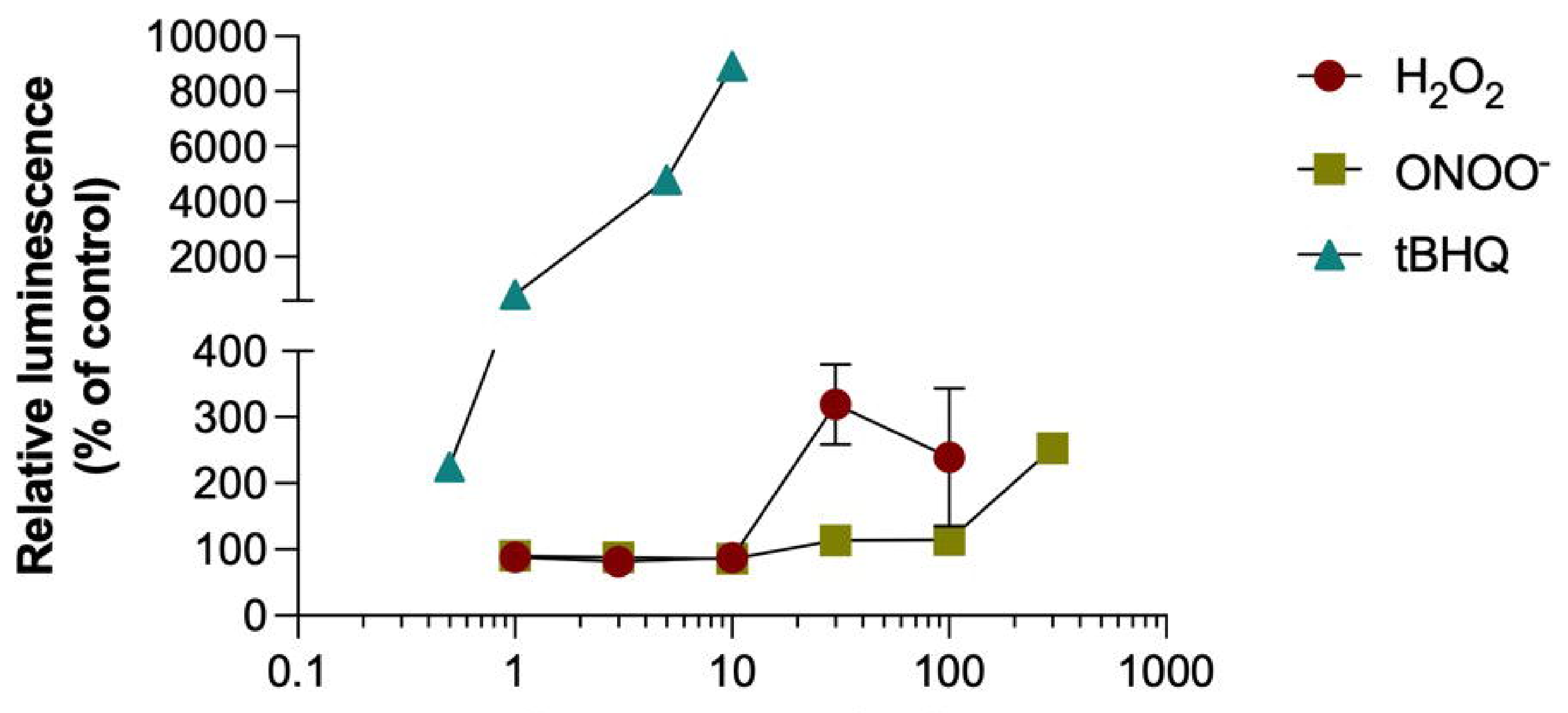

Concentration $(\mu \mathrm{M})$ 